\title{
Energy-Robustness Tradeoff in Cellular Network Power Control
}

\author{
Chee Wei Tan, Member, IEEE, Daniel P. Palomar, Member, IEEE, and Mung Chiang, Senior Member, IEEE
}

\begin{abstract}
In the seminal paper by Foschini and Miljanic in 1993, a distributed power control algorithm was developed to meet SIR targets with minimal powers in cellular network uplinks. Since the SIR on an active link may dip below the SIR target during the transient after a new user enters the cell, Bambos et al. proposed an active link protection algorithm to provide robustness, at the expense of higher energy consumption. This paper examines the tradeoff between energy and robustness. An optimization problem is formulated where robustness is captured in the constraint and the price of robustness penalized in the objective function. A distributed algorithm is developed to solve this problem. Local convergence and optimality of equilibrium are proved for the algorithm. The objective function modulates the tradeoff between energy and robustness, and between energy and speed of admission, as illustrated through a series of numerical experiments. A parameterized family of objective functions is constructed to control the transient and equilibrium properties of robust distributed power control.
\end{abstract}

Index Terms-Distributed optimization, duality, power control, wireless networks.

\section{INTRODUCTION}

$\mathbf{P}$ OWER control on the uplinks of an interference-limited cellular network serves two major roles: managing energy consumption and managing signal interference. These two roles can be conflicting, and it is their tradeoff that we study in this paper. Controlling signal-to-interference ratio (SIR) is the key to providing quality-of-service to the mobile users, and power control is often used to attain desired SIRs and has been extensively studied since the early 1990s. In particular, the Distributed Power Control (DPC) algorithm in [1] by Foschini and Miljanic has been widely used and extended (e.g., in [2]-[9]). Following a simple power update rule, Algorithm DPC converges to a minimal power vector that can attain a fixed SIR target vector, provided it is feasible. While the equilibrium of Algorithm DPC is well-understood, what happens during the transient is more challenging to characterize and much less clear. In particular, the SIR of an active link can easily dip below the SIR targets when there is a slight perturbation such as the entry of a new user.

Manuscript received September 01, 2007; revised April 13, 2008, and June 25, 2008; approved by IEEE/ACM TRANSACTIONS ON NETWORKING Editor S. Borst. First published October 03, 2008; current version published June 17, 2009. This work was supported in part by the National Science Foundation under NSF CCF-0448012 and NSF CNS-0720570, the Air Force Office of Scientific Research under AFOSR FA9550-06-1-0297, and the Office of Naval Research under ONR N00014-07-1-0864. This paper was presented in part at the IEEE INFOCOM 2007, Anchorage, AK.

C. W. Tan and M. Chiang are with the Department of Electrical Engineering, Princeton University, Princeton NJ 08544 USA (e-mail: cheetan@princeton. edu).

D. P. Palomar is with the Department of Electronic and Computer Engineering, Hong Kong University of Science and Technology, Clear Water Bay, Kowloon, Hong Kong.

Color versions of one or more of the figures in this paper are available online at http://ieeexplore.ieee.org.

Digital Object Identifier 10.1109/TNET.2008.2003336

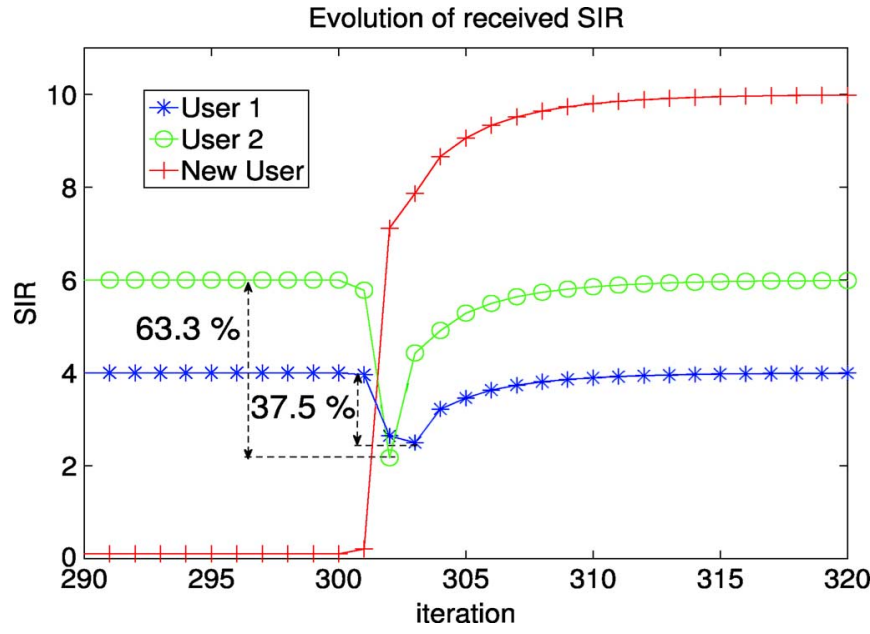

Fig. 1. A small numerical example of using Algorithm DPC in a CDMA cell. Originally, there are two active users, but a new user enters the same channel at the 300th time slot. The received SIRs of the two active users experience an SIR outage that deviates from the SIR targets by as much as $37 \%$ and $63 \%$ during the transient.

Consider the following simple example of a small CDMA cell originally with two active users. A new user enters using Algorithm DPC, and causes an SIR outage that deviates from the SIR targets by as much as $63 \%$ during the transient that lasts for approximately 20 time slots, as shown in Fig. 1. This simple example illustrates that a key limitation of Algorithm DPC is the lack of guaranteed signal quality in times of congestion.

How to prevent such dips, and in general, how to admit users and manage congestion in cellular network power control becomes an important question. In particular, to guarantee that active links continue to have acceptable received SIR when there are disturbances in the network, an active link protection scheme has to be incorporated with access control. In [2], the algorithm of DPC with Active Link Protection (DPC/ALP) was developed by Bambos et al. In [3], the DPC/ALP algorithm was further developed for both voice and data communication over wireless ad-hoc networks. First, an active link protection scheme provides a protection margin $\epsilon$ to the SIR targets of existing users. Second, new users ramp up their power following a different rule, one that avoids creating new interference too fast.

Intuitively, the protection margin $\epsilon$ has to be large enough such that the received SIRs of active link connections do not drop below the minimum SIR requirements and cause SIR outage. On the other hand, it should not be too large, otherwise this might lead to excessive power expenditure. How to control this energy-robustness tradeoff is one of the motivations for this paper. We use the term "robust" in the sense of safety margin against SIR outage. A related issue is the transient behavior of Algorithm DPC before it reaches an equilibrium, especially the tradeoff between quick admittance of new users to their SIR 
targets and low interference created during the admission. In both questions, the intuition is that $\epsilon$ should be time-varying instead of a constant, controlled possibly by some "interference price" that is updated by inferring the network congestion level from local measurements. Such an intuition is made rigorous in this paper, and open questions on the transient behaviors of power control answered consequently.

After reviewing the DPC and DPC/ALP algorithms in Section II, we formulate an optimization problem in Section III-A, where robustness is captured in the constraint and the price of robustness penalized in the objective function. Then in Section III-B, we present a distributed algorithm that solves this problem. The development of this algorithm, the Robust DPC (RDPC) algorithm is shown in Section IV. A parameterized family of objective functions is constructed to control the transient and equilibrium properties of robust distributed power control, as shown in Section V. The concepts of congestion measure and interference price are further quantified in the same section. Convergence of the algorithm and optimality of equilibrium are proved for Algorithm RDPC (Theorems 1 and 3). The effectiveness of modulating the tradeoff between energy and robustness, and between interference management and speed of convergence, is demonstrated through a series of numerical experiments in Section VI, before we conclude in Section VII. To make the flow of presentation smoother, we collect all the proofs in the Appendix.

The following notation is used. Boldface uppercase letters denote matrices, boldface lowercase letters denote column vectors, italics denote scalars, and $\mathbf{x} \succeq \mathbf{y}$ denotes componentwise inequality between vectors $\mathbf{x}$ and $\mathbf{y}$.

\section{REVIEW: DPC AND DPC/ALP}

\section{A. Algorithm DPC}

Consider a CDMA cellular wireless network with $L$ logical links (equivalently, transceiver pairs), which models a single cell of a CDMA system. Our analysis also applies more generally to a multi-cell CDMA system, where a base station in each cell acts as a central coordinator of uplink resource allocation. The transmit power for the $l$ th link is denoted by $p_{l}$ for $l=1, \ldots, L$. Assuming a matched-filter receiver, the SIR for the receiver on link $l$ can be written as [10]

$$
\operatorname{SIR}_{l}(\mathbf{p})=\frac{G_{l l} p_{l}}{\sum_{j \neq l} G_{l j} p_{j}+n_{l}}
$$

where $G_{l j}$ are the channel gains from transmitter $j$ to receiver $l$, and $n_{l}$ is the additive white Gaussian noise (AWGN) power for receiver $l$. The processing gain of the CDMA system is assumed to be absorbed into $G_{l l}$ in (1) for all $l$ [10]. We also assume that the channel is frequency-flat and is constant for the duration of a transmission, e.g., under slow fading.

The following power control problem of minimizing the total transmitted power subject to SIR constraints was studied in [1] and [11]:

$$
\begin{aligned}
\text { minimize } & \sum_{l} p_{l} \\
\text { subject to } & \operatorname{SIR}_{l}(\mathbf{p}) \geq \gamma_{l} \quad \forall l, \\
\text { variables : } & p_{l} \quad \forall l .
\end{aligned}
$$

The SIR constraint is the requirement that the $l$ th received SIR is above a given SIR target $\gamma_{l}$ for all $l$. Problem (2) can be rewritten as a linear program in matrix form:

$$
\begin{aligned}
\text { minimize } & \mathbf{1}^{\top} \mathbf{p} \\
\text { subject to } & (\mathbf{I}-\mathbf{F}) \mathbf{p} \succeq \mathbf{v}
\end{aligned}
$$

where $\mathbf{I}$ is the identity matrix, $\mathbf{F}$ is the matrix with entries [1]

$$
F_{i j}= \begin{cases}0, & \text { if } i=j \\ \frac{G_{i j} \gamma_{i}}{G_{i i}}, & \text { if } i \neq j\end{cases}
$$

and

$$
\mathbf{v}=\left(\frac{\gamma_{1} n_{1}}{G_{11}}, \frac{\gamma_{2} n_{2}}{G_{22}}, \ldots, \frac{\gamma_{L} n_{L}}{G_{L L}}\right)^{\top} .
$$

The algorithm proposed by Foschini and Miljanic is given by [1]

$$
\mathbf{p}(k+1)=\mathbf{F} \mathbf{p}(k)+\mathbf{v}
$$

where $k$ indexes discrete time slots. It converges asymptotically to $\mathbf{p}^{\star}$, a solution to (2), whenever it exists, i.e., when the fixed SIR targets are feasible. Feasibility of SIR targets is well-known to be equivalent to the following condition: the Perron-Frobenius eigenvalue of $\mathbf{F}$, denoted as $\rho(\mathbf{F})$, is strictly less than 1 .

Re-writing (5) in scalar form, it is clear that (2) can be solved using the following distributed power control (DPC) algorithm:

$$
p_{l}(k+1)=\frac{\gamma_{l}}{\operatorname{SIR}_{l}(\mathbf{p}(k))} p_{l}(k) \quad \forall l
$$

where $\operatorname{SIR}_{l}(\mathbf{p}(k))$ is the received SIR at the $k$ th time slot. The update in (6) is distributed as each user only needs to monitor its individual received SIR and can update by (6) independently and asynchronously [9]. Intuitively, each user $l$ increases its power when its $\operatorname{SIR}_{l}(\mathbf{p}(k))$ is below $\gamma_{l}$ and decreases it otherwise, so that $\operatorname{SIR}_{l}(\mathbf{p}(k))$ in the next time slot is exactly the target $\gamma_{l}$ if no other users change their powers. Furthermore, the optimal power allocation $\mathbf{p}^{\star}$ in (2) is achieved in the limit as $k \rightarrow \infty$, and satisfies $(\mathbf{I}-\mathbf{F}) \mathbf{p}^{\star}=\mathbf{v}$, i.e., the constraints in (3) are tight at optimality. Algorithm DPC is widely used for its simplicity and effectiveness, and can be interpreted from several angles, e.g., best response strategy of a game, or linear update for a fixed-point equation [9].

\section{B. Algorithm DPC/ALP}

By extending the Foschini-Miljanic power control scheme, Bambos et al. [2] proposed the Distributed Power Control with Active Link Protection (DPC/ALP) algorithm to protect active users from new users that access the same channel. The two key ideas of the DPC/ALP algorithm are: 1) the gradual power-up of new users; and 2) the introduction of an SIR margin $\epsilon$ to cushion the existing users, which is accomplished by modifying the SIR constraint in (2) to

$$
\operatorname{SIR}_{l}(\mathbf{p}) \geq \gamma_{l}(1+\epsilon) \quad \forall l
$$

where $\epsilon>0$. Clearly, the parameter $\epsilon$ serves as a protection margin for users that are running (6) and helps keep them from falling below $\boldsymbol{\gamma}$ when new users access the same channel.

Specifically, for a given $\epsilon$, Algorithm DPC/ALP is given by

$$
p_{l}(k+1)=\left\{\begin{array}{ll}
\frac{(1+\epsilon) \gamma_{l}}{\operatorname{SIR}_{l}(\mathbf{p}(k))} p_{l}(k), & \text { if } \operatorname{SIR}_{l}(\mathbf{p}(k)) \geq \gamma_{l} \\
(1+\epsilon) p_{l}(k), & \text { if } \operatorname{SIR}_{l}(\mathbf{p}(k))<\gamma_{l}
\end{array} \quad \forall l .\right.
$$


Implicitly, the DPC/ALP algorithm solves the following problem of power minimization subject to a fixed robustness setting:

$$
\begin{aligned}
\operatorname{minimize} & \sum_{l} p_{l} \\
\text { subject to } & \operatorname{SIR}_{l}(\mathbf{p}) \geq \gamma_{l}(1+\epsilon) \quad \forall l, \\
\text { variables : } & p_{l} \quad \forall l .
\end{aligned}
$$

With the $\epsilon$ fixed, (9) can still be rewritten as a linear program in a way similar to (3), and Algorithm DPC/ALP converges to the optimal solution of (9) if and only if $(1+\epsilon) \rho(\mathbf{F})<1$ [2].

A larger $\epsilon$ obviously provides more protection to existing users, but comes at a price of higher power expenditure. Furthermore, a larger $\epsilon$ makes user admission faster, but may cause excessive interference or even infeasibility of the enhanced target SIR. How to control these two tradeoffs? Our intuitive design is that $\epsilon$ should be time-varying instead of being a constant, controlled possibly by some "interference price" that is updated by inferring the network congestion level from local measurements. This intuition will be made clear in the rest of the paper.

\section{Robust Power Control: Problem AND Algorithms}

To effectively control the tradeoffs and to accommodate engineering requirements such as rise-over-thermal constraints and convergence speed, Algorithm RDPC is developed based on sensitivity analysis of the effects of power changes, on interference prices obtained from Lagrangian duality theory, on congestion measures related to the size of the Perron-Frobenius eigenvalue, and on a primal-dual update equation with desirable convergence properties. It turns out that Algorithm RDPC solves an underlying optimization problem, whose objective function can be tuned to influence the behavior of Algorithm RDPC in a predictive way. To make the presentation flow from the first principles, we will first present the underlying optimization problem and Algorithm RDPC in this section, before we show how Algorithm RDPC is developed and its properties proved in Section IV. We then discuss the application of Algorithm RDPC for energy-robustness tradeoff in Section V.

\section{A. Robust Power Control Problem Formulation}

We first present a general problem formulation for the uplink transmission in a CDMA cell that takes robustness into account. Using the protection margin in (7) to enhance the SIR constraints, we consider minimizing the total power expenditure plus a cost function, over both $\mathbf{p}$ and $\epsilon$ :

$$
\begin{aligned}
\text { minimize } & \sum_{l} p_{l}+\phi(\epsilon) \\
\text { subject to } & \operatorname{SIR}_{l}(\mathbf{p}) \geq \gamma_{l}(1+\epsilon) \quad \forall l, \\
& \epsilon \geq 0, p_{l} \geq 0 \quad \forall l, \\
\text { variables : } & \epsilon, p_{l} \quad \forall l
\end{aligned}
$$

where now $\epsilon$ is an optimization variable and $\phi(\epsilon)$ is a decreasing, convex, twice differentiable cost function that captures the tradeoff in adjusting $\epsilon$. This objective function will be very useful in modulating the tradeoff between robustness and energy both at equilibrium and during the transient.
The following necessary condition that characterizes any feasible solution to (10) will become useful in later developments and proofs:

Lemma 1: If $\mathbf{p}$ and $\epsilon$ are feasible in problem (10), then

$$
(1+\epsilon) \rho(\mathbf{F})<1 .
$$

We note that (10) can no longer be rewritten as a linear program as can be done with (2) and (9), and in fact it is a nonconvex optimization problem. It can nevertheless be rewritten as a convex optimization problem for certain functions $\phi(\epsilon)$. First, we apply a $\log$ transformation to $p_{l}$, for all $l$, and $\epsilon(\tilde{p}=\log p$ and $\tilde{\epsilon}=\log \epsilon$ ), obtaining the following equivalent problem:

$$
\begin{aligned}
\operatorname{minimize} & \sum_{l} e^{\tilde{p}_{l}}+\phi\left(e^{\tilde{\epsilon}}\right) \\
\text { subject to } & \log \left(\operatorname{SIR}_{l}(\tilde{\mathbf{p}}) / \gamma_{l}\right) \geq \log \left(1+e^{\tilde{\epsilon}}\right) \quad \forall l, \\
\text { variables: } & \tilde{p}_{l} \quad \forall l, \tilde{\epsilon} .
\end{aligned}
$$

We will later focus on those $\phi$ satisfying the condition in the following.

Lemma 2: The optimization problem in (12) is convex if $\left(\partial^{2} \phi(z) / \partial z^{2}\right) z+\partial \phi(z) / \partial z \geq 0$ for $z>0$.

We will show in Section IV that designing appropriate $\phi$ can be used to control the power of each user both at equilibrium and during transient, and we look at $\phi$ that characterizes the energy-robustness tradeoff.

\section{B. RDPC Algorithm}

We now give the main algorithm: Robust Distributed Power Control (RDPC). The details of its derivation will be given in the next sections (in particular, (14), (15) follow from Theorem 2 and (16) follows from Theorem 1). Computing the optimal $\mathbf{p}$ and $\epsilon$ in (10) by Algorithm RDPC is aided by an "interference price" vector $\boldsymbol{\nu}$, which is key to access the impact of $\epsilon$ on the increase in the total power consumption $\left(\sum_{l} p_{l}\right.$ in (10)), as shown in Section IV-A. It will also be shown that $\boldsymbol{\nu}$ is the Lagrange multiplier vector for (12).

\section{Algorithm RDPC:}

- The base station initiates at $\epsilon(0)$. New users power up with sufficiently small $p_{l}(0)$, e.g., $p_{l}(0)=n_{l}$.

Update by each user $l$ :

- Update the transmitter power $p_{l}(k+1)$ at the $(k+1)$ th step:

$$
p_{l}(k+1)= \begin{cases}\frac{(1+\epsilon(k)) \gamma_{l}}{\operatorname{SIR}_{l}(\mathbf{p}(k))} p_{l}(k), & \text { if } \operatorname{SIR}_{l}(\mathbf{p}(k)) \geq \gamma_{l} \\ (1+\epsilon(k)) p_{l}(k), & \text { if } \operatorname{SIR}_{l}(\mathbf{p}(k))<\gamma_{l}\end{cases}
$$

\section{Update by the base station:}

- Update auxiliary variable $\mathbf{x}(k+1)$ :

$$
\mathbf{x}(k+1)=(1+\epsilon(k)) \mathbf{F}^{\top} \mathbf{x}(k)+\mathbf{1}
$$

- Update interference price $\boldsymbol{\nu}(k+1)$ :

$$
\nu_{l}(k+1)=x_{l}(k+1) p_{l}(k+1) \quad \forall l
$$




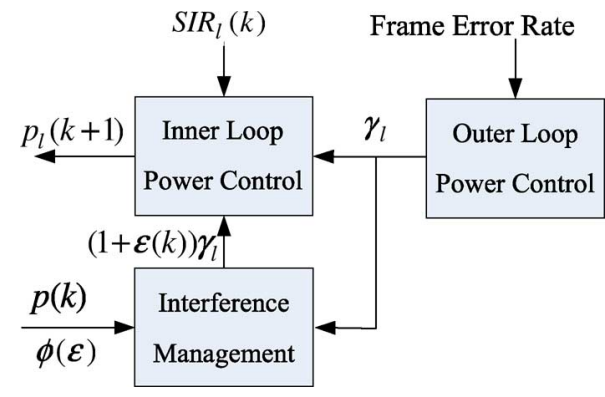

Fig. 2. Schematic of Algorithm RDPC for uplink power control.

- Update protection margin $\epsilon(k+1)$ by solving

$$
-\left.\frac{\partial \phi(\epsilon)}{\partial \epsilon}\right|_{\epsilon=\epsilon(k+1)}(1+\epsilon(k+1))=\mathbf{1}^{\top} \boldsymbol{\nu}(k+1) .
$$

Note that the power update (13) differs from the DPC/ALP algorithm in [2] in that $\epsilon$ is not a constant parameter as in [2], but is updated at each time slot according to variables $\mathbf{x}$ and $\boldsymbol{\nu}$. Interestingly, (16) quantifies the remark on choosing the parameter $\epsilon$ in [2]: " $\epsilon$ should be chosen such that $(1+\epsilon)$ is larger when the network is uncongested, so that links power up fast, and grow smaller as congestion builds up to have links power up more gently".

Also note that both the DPC and DPC/ALP algorithms are primal algorithms with linear updates, whereas Algorithm RDPC is a primal-dual algorithm with nonlinear updates.

A general schematic diagram of Algorithm RDPC for an uplink transmission is shown in Fig. 2. The inner loop power control block consists of a transmit power update for each user based on the measured SIR values [cf. (13)], and the outer loop power control block adjusts the SIR targets $\gamma$ as a function of the frame error rates. ${ }^{1}$ The interference management block takes as input the measured power of all users and $\boldsymbol{\gamma}$, and, based on a particular cost function $\phi(\epsilon)$, modulates the parameter $\epsilon$, which is then fed to the inner loop block [cf. (14)-(16)].

As proved in Appendix C, we have the following theorem for Algorithm RDPC that connects it to the underlying optimization model, and a stronger version for a special case will be presented later as Theorem 3.

Theorem 1: If $\{\epsilon(k)\}$ converges to a finite value $\epsilon^{\star}$, and $\rho(\mathbf{F})<1 /\left(1+\epsilon^{\star}\right)$, then Algorithm RDPC converges to a global optimum $\left(\mathbf{p}^{\star}, \epsilon^{\star}\right)$ of problem (10).

\section{DEVElopment AND IMPLEMENTATION OF RDPC}

\section{A. Step 1: Sensitivity Analysis}

Intuitively, the amount of congestion in the system and the "price" of maintaining the SIR constraints should be a factor in determining how robust a power control algorithm needs to be. A quantitative study in this section, based on a sensitivity analysis of the Foschini-Miljanic power control problem in [12], will make this intuition precise. We first consider the case of $\epsilon=0$ in (10), which corresponds to (2). We then use sensitivity analysis to show how adjusting $\gamma$ affects the solution for (2), which

\footnotetext{
${ }^{1}$ The inner and outer loop power control blocks exist in current CDMA cellular network. Implementing Algorithm RDPC thus entails only a small modification by incorporating an interference management block as shown in Fig. 2.
}

further instructs us on how to choose the right cost function $\phi(\epsilon)$ in (10).

Recall that the SIR constraints in (2) are tight at optimality, assuming that there is a feasible power allocation for all users. Hence, tightening or loosening this constraint set affects the optimal value of (2). The following result relates the sensitivity of the SIR constraint and the total power requirement in (2).

First, for the $l$ th SIR constraint in (2), we define a perturbed SIR target $\gamma_{l} / u_{l}$ where $1 / u_{l}$ represents a fractional perturbation of the SIR target $\gamma_{l}$, and substitute the $l$ th SIR constraint in (2) by $\gamma_{l} / \operatorname{SIR}_{l}(\mathbf{p}) \leq u_{l}$ for all $l$. We have $0<u_{l}<1$ or $u_{l}>1$ if we tighten or loosen the lth SIR constraint, respectively. Next, we define $f^{\star}(\mathbf{u})$ as the optimal value of (2) with these perturbed constraints:

$$
f^{\star}(\mathbf{u})=\inf \left\{\sum_{l} p_{l} \mid \gamma_{l} / \operatorname{SIR}_{l}(\mathbf{p}) \leq u_{l} \forall l\right\} .
$$

If $f^{\star}(\mathbf{u})$ does not exist for some $\mathbf{u}$, we define $f^{\star}(\mathbf{u})=\infty$. Although the optimization problem in (17) can be rewritten as a linear program in $\mathbf{p}$, it is expressed in a form that allows us to apply a change-of-variable technique, which is a key step that leads to RDPC. Let $\tilde{p}_{l}=\log p_{l}$ and the parameter $\tilde{u}_{l}=$ $\log u_{l}$, and taking the logarithm of the SIR constraints, we write $f^{\star}(\mathbf{u})$ in the log transformed parameter $\tilde{\mathbf{u}}$ as $\tilde{f}^{\star}(\tilde{\mathbf{u}})$, and $\tilde{f}^{\star}(\tilde{\mathbf{u}})$ is determined by solving the following problem:

$$
\begin{aligned}
\text { minimize } & \sum_{l} e^{\tilde{\tilde{l}}_{l}} \\
\text { subject to } & \log \left(\gamma_{l} / \operatorname{SIR}_{l}(\tilde{\mathbf{p}})\right) \leq \tilde{u}_{l} \quad \forall l, \\
\text { variables : } & \tilde{p}_{l} \quad \forall l .
\end{aligned}
$$

The following result quantifies the tradeoff between power expenditure and robustness [13].

Lemma 3: Let $\nu_{l}^{\star}$, for all $l$, be the optimal Lagrange multipliers of the unperturbed problem in (18), (i.e., for $\tilde{\mathbf{u}}=\mathbf{0}$ ), then

$$
100 \frac{\tilde{f}^{\star}\left(\frac{\beta_{l}}{100} \mathbf{e}_{l}\right)-\tilde{f}^{\star}(\mathbf{0})}{\tilde{f}^{\star}(\mathbf{0})}=-\beta_{l} \nu_{l}^{\star} / \tilde{f}^{\star}(\mathbf{0})+o\left(\beta_{l}\right)
$$

where $\mathbf{e}_{l}$ is a vector that has all its entries 0 except the $l$ th entry which is 1 .

The engineering implication is as follows. Relaxing (or tightening) the $l$ th SIR target constraint by $\beta_{l}$ percent in (2) decreases (or increases) the total power by approximately $\beta_{l} \nu_{l}^{\star} / \tilde{f}^{\star}(\mathbf{0})$ percent, for a small $\beta_{l}$. Hence, the total power reduction (or increment) is approximately $\sum_{l} \beta_{l} \nu_{l}^{\star} / \tilde{f}^{\star}(\mathbf{0})$ percent. Fig. 3 shows the supporting hyperplane at $f^{\star}(\mathbf{0})$ with gradient $-\nu_{l}^{\star}$ [13], and illustrates Lemma 3. If a user with large $\nu_{l}^{\star}$ compromises the target SIR slightly, all users obtain both considerable power saving and lower interference simultaneously.

Example 1: As an illustrative numerical example validating Lemma 3, we compare the results given by Lemma 3 with those in the simulation reported in [14] for a network with three users. From initial SIR targets $(7,7,7)^{\top}$ (in $\mathrm{dB}$ ), only Users 2 and 3 change their SIR targets, i.e., from $7 \mathrm{~dB}$ to $6.90 \mathrm{~dB}$ (first operating point) and then to $6.50 \mathrm{~dB}$ (second operating point).

The $\boldsymbol{\nu}^{\star}$ (see Theorem 2 later for its computation) that corresponds to the network operating at SIR targets $(7,6.9,6.9)^{\top}$ and $(7,6.5,6.5)^{\top}$ (in $\mathrm{dB}$ ) are, respectively, $(128.680,155.598,107.532)^{\top}$ and $(8.529,10.209,7.066)^{\top}$ 


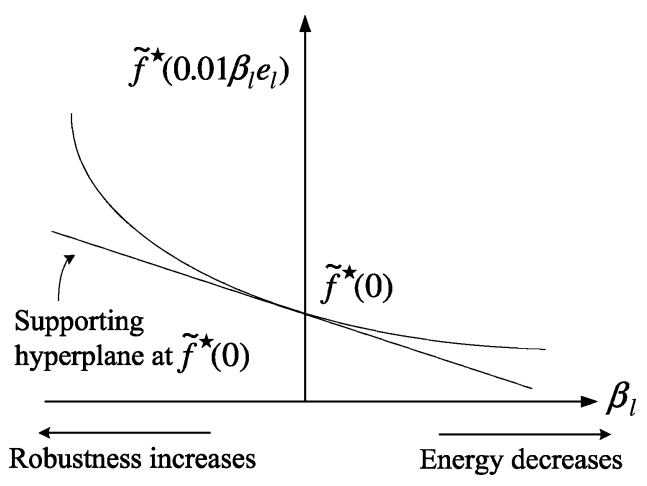

Fig. 3. Robustness-energy tradeoff using sensitivity analysis.

The numerical percent decrease in total power, as reported in [14], when the SIR operating point changes to the first operating point and when the SIR operating point changes to the second operating point is given by $64.600 \%$ and $75.200 \%$, respectively.

Now, using $\boldsymbol{\nu}^{\star}$, the prediction given by Lemma 3 when the SIR operating point changes to the first operating point and when the SIR operating point changes to the second operating point is given by $64.650 \%$ and $74.483 \%$, respectively. The prediction of Lemma 3 is observed to be quite accurate, and the numerical experiment in [14] verifies the theory very well.

\section{B. Step 2: Basic Primal-Dual Algorithm}

In this subsection, we will show how to compute $\nu$ in Lemma 3 , and then develop the iterative updates used by Algorithm RDPC in Section III-B.

Theorem 2: The optimal power $\mathbf{p}^{\star}$ in (2) and the Lagrange multiplier $\boldsymbol{\nu}^{\star}$ in the unperturbed problem in (18) satisfy

$$
\nu_{l}^{\star}=p_{l}^{\star}\left(1+\sum_{i \neq l} \frac{G_{i l} \nu_{i}^{\star}}{\sum_{j \neq i} G_{i j} p_{j}^{\star}+n_{i}}\right) \quad \forall l .
$$

Furthermore, the following iterative updates can be used to compute $\boldsymbol{\nu}^{\star}$ :

$$
\begin{aligned}
& \mathbf{p}(k+1)=\mathbf{F} \mathbf{p}(k)+\mathbf{v}, \\
& \mathbf{x}(k+1)=\mathbf{F}^{\top} \mathbf{x}(k)+\mathbf{1},
\end{aligned}
$$

and

$$
\nu_{l}(k+1)=x_{l}(k+1) p_{l}(k+1) \quad \forall l .
$$

As $k \rightarrow \infty, \nu_{l}(k)$ converges to $\nu_{l}^{\star}$ in (20) for all $l$ if and only if $\rho(\mathbf{F})<1$.

In Theorem 2, $\nu_{l}^{\star}=x_{l}^{\star} p_{l}^{\star}$ for all $l$. While $\mathbf{p}$ are the primal variables in (2) and $\boldsymbol{\nu}$ are the dual variables of the unperturbed problem in (18), $\mathbf{x}$ are the auxiliary variables assisting with the computation of the primal and dual variables. It is interesting to note that $\mathbf{x}^{\star}$ is also the optimal solution to the following optimization problem:

$$
\begin{aligned}
\operatorname{maximize} & \mathbf{v}^{\top} \mathbf{x} \\
\text { subject to } & \left(\mathbf{I}-\mathbf{F}^{\top}\right) \mathbf{x} \preceq \mathbf{1},
\end{aligned}
$$

which is the Lagrange dual problem of (3). Using the iterations (21)-(23), as long as $\mathbf{p}(k)$ and $\mathbf{x}(k)$ are feasible in (3) and (24), respectively, then $\log (\mathbf{p}(k))$ and $\boldsymbol{\nu}(k)$ are primal and dual feasible with respect to (18), respectively.
Finally, we remark that there is an interesting duality between the algorithm computing $\mathbf{p}^{\star}$ and that computing $\mathbf{x}^{\star}$ :

Algorithmic Duality Correspondence

$$
\begin{array}{ccc}
\text { columns of } \mathbf{F} & \leftrightarrow & \text { rows of } \mathbf{F} \\
\mathbf{1} & \leftrightarrow & \mathbf{v} \\
\mathbf{x}^{\star} \text { with } \nu_{l}^{\star}=x_{l}^{\star} p_{l}^{\star} & \leftrightarrow & \mathbf{p}^{\star} \\
\mathbf{v}^{\top} \mathbf{X}^{\star} & \leftrightarrow & \mathbf{1}^{\top} \mathbf{p}^{\star}
\end{array}
$$

\section{Step 3: Further Simplifications}

Note that (21) can be computed distributively just like in Algorithm DPC (6), but (22) cannot be computed distributively as each user needs the cross-channel gains in (22). However, by exploiting the uplink-downlink duality in [6], even (22) can be carried out distributively in a time division duplex (TDD) network, where the auxiliary variable $\mathbf{x}$ in Algorithm RDPC takes on a physical interpretation as a virtual downlink power, as will be shown below. The downlink case refers to transmission from the base station to the users with the same SIR target as the uplink case. In this case, the channel matrix is given by $\mathbf{G}^{\top}$. The update of $\epsilon$ in (16) must still be done centrally at the base station, which acts as the central coordinator for uplink resource allocation for each cell in a multi-cell CDMA system.

First, the fact that all the constraints in (24) are tight at optimality implies that the optimizer to (24) is equivalent to the optimizer of the following problem:

$$
\begin{aligned}
\operatorname{minimize} & \mathbf{1}^{\top} \mathbf{x} \\
\text { subject to } & \left(\mathbf{I}-\mathbf{F}^{\top}\right) \mathbf{x} \succeq \mathbf{1}
\end{aligned}
$$

which can be interpreted as minimizing the total power for the downlink case (with $\mathbf{x}$ having a physical interpretation as the downlink power) but with AWGN power $G_{l l} / \gamma_{l}$ for all $l$ [6]. Compare (25) with the uplink case in (3). As in [6], the next step to a distributed Algorithm RDPC is to create a virtual downlink power minimization, which is solved by (25). Note that the virtual downlink problem does not correspond to the real downlink power control problem, but acts as an intermediary mechanism for message passing in the downlink phase. The virtual downlink problem corresponds to the real downlink power control problem only if the real downlink AWGN noise power for each link is exactly $G_{l l} / \gamma_{l}$ for all $l$.

Therefore, (14) in Algorithm RDPC is rewritten as

$$
x_{l}(k+1)=\frac{(1+\epsilon(k)) \gamma_{l}}{\operatorname{SiR}_{l}(\mathbf{x}(k))} x_{l}(k) \quad \forall l
$$

where $\mathrm{SIIR}_{l}(\mathbf{x}(k))$ is the virtual downlink received SIR:

$$
\operatorname{SîR}_{l}(\mathbf{x}(k))=\frac{G_{l l} x_{l}(k)}{\sum_{j \neq l} G_{j l} x_{j}(k)+G_{l l} /\left(\gamma_{l}(1+\epsilon(k))\right)} \quad \forall l .
$$

Unlike (1), which is a scalar measurement output at the receiver, (27) has to be computed by keeping separate copies of the received virtual powers $G_{j l} x_{j}(k)$ for all $j$ at the $l$ link during the downlink time slot. 


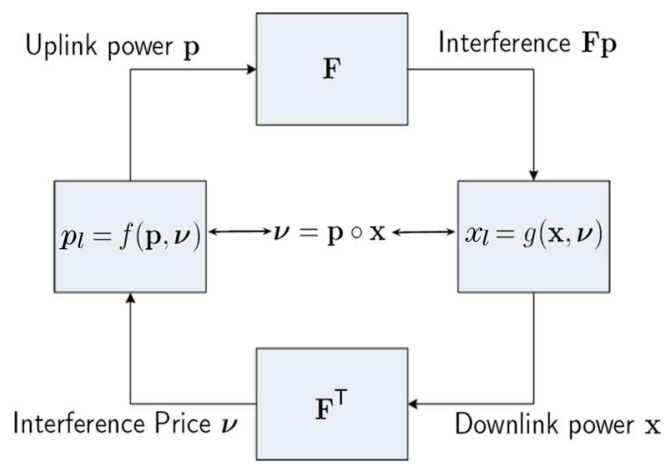

Fig. 4. Algorithm RDPC and uplink-downlink duality. The functions $f$ and $g$ are the updates for the uplink power $\mathbf{p}$ and the downlink power $\mathbf{x}$, respectively. The interference price $\boldsymbol{\nu}$ is the Schur product of $\mathbf{p}$ and $\mathbf{x}$, i.e., $\nu_{l}=p_{l} x_{l}$.

Thus, (13) and (14), (15) in Algorithm RDPC correspond to the uplink and (virtual) downlink case, respectively, and we can interpret $\nu_{l}$ as the product of the uplink and downlink power of the $l$ th user. Fig. 4 illustrates the role of uplink-downlink duality in Algorithm RDPC. The modified Algorithm RDPC for a TDD cellular network is given as follows, where the base station only needs to compute and broadcast $\epsilon$.

Algorithm RDPC (for TDD Cellular Network):

- The base station initiates at $\epsilon(0)$. New users power up with sufficiently small $p_{l}(0)$, e.g., $p_{l}(0)=n_{l}$.

\section{Update by each user $l$ during the uplink time slot:}

- Update the transmitter power $p_{l}(k+1)$ at the $(k+1)$ th step:

$$
p_{l}(k+1)= \begin{cases}\frac{(1+\epsilon(k)) \gamma_{l}}{\operatorname{SIR}_{l}(\mathbf{p}(k))} p_{l}(k), & \text { if } \operatorname{SIR}_{l}(\mathbf{p}(k)) \geq \gamma_{l} \\ (1+\epsilon(k)) p_{l}(k), & \text { if } \operatorname{SIR}_{l}(\mathbf{p}(k))<\gamma_{l}\end{cases}
$$

\section{Update by each user $l$ during the downlink time slot:}

- Update the virtual transmitter power $x_{l}(k+1)$ at the $(k+1)$ th step:

$$
x_{l}(k+1)=\frac{(1+\epsilon(k)) \gamma_{l}}{\operatorname{SîR}_{l}(\mathbf{x}(k))} x_{l}(k)
$$

- Update the Lagrange multiplier $\nu_{l}(k+1)$ :

$$
\nu_{l}(k+1)=x_{l}(k+1) p_{l}(k+1) .
$$

\section{Update by the base station:}

- Update the protection margin $\epsilon(k+1)$ by solving

$$
-\left.\frac{\partial \phi(\epsilon)}{\partial \epsilon}\right|_{\epsilon=\epsilon(k+1)}(1+\epsilon(k+1))=\mathbf{1}^{\top} \boldsymbol{\nu}(k+1) \text {. }
$$

\section{BALANCING THE TRADEOFFS}

Recall that there are two main tradeoffs: one between robustness and energy at equilibrium, and another one between the speed of admission and energy during transience. Both tradeoffs can be controlled by changing the curvature of the function $\phi(\epsilon)$, which then changes the dynamic and equilibrium properties of Algorithm RDPC. These two tradeoffs are discussed in the first two subsections, followed by a discussion on "interference price" in Algorithm RDPC and "congestion measure" in a cellular network in the last subsection.

\section{A. Choosing $\phi(\epsilon)$}

Based on the sensitivity analysis result in Lemma 3, the extra power needed to provide $\epsilon^{\star}$ amount of SIR margin is

$$
100\left(\mathbf{1}^{\top} \boldsymbol{\nu}^{\star} \epsilon^{\star} / \mathbf{1}^{\top} \mathbf{p}^{\star}\right)
$$

percent (see Appendix F), or, from (16),

$$
-\left.\frac{\partial \phi(\epsilon)}{\partial \epsilon}\right|_{\epsilon=\epsilon^{\star}} 100\left(1+\epsilon^{\star}\right) \epsilon^{\star} / \mathbf{1}^{\top} \mathbf{p}^{\star}
$$

percent. For example, a network operator with a good estimate of (33) may choose to not admit new users because that might double the power expenditure with just 3\% safety margin, as in the example in Section IV-A. Similarly, substantial energy savings could be obtained if the target SIR or protection margin is reduced a little.

Suppose the network can tolerate at most an increase of $100 \delta / \mathbf{1}^{\top} \mathbf{p}^{\star}$ percent in total power to limit interference. This $\delta$ can be obtained from different kinds of models. For example, in [15], the authors propose an in-cell rise-over-thermal (IROT) constraint. Given an IROT constraint, $\delta$ can be configured as the input parameter for Algorithm RDPC. Now, using this upper bound $\delta$ and (33), we have

$$
\frac{\partial \phi(\epsilon)}{\partial \epsilon}=-\frac{\delta}{\epsilon(1+\epsilon)}
$$

which upon integration yields

$$
\phi(\epsilon)=\delta \log (1+1 / \epsilon) .
$$

It is easy to verify that $\phi(\epsilon)$ is strictly convex, decreasing, and satisfies Lemma 2, thus can be used in Algorithm RDPC. Alternatively, an upper bound on the relative change $\delta / \mathbf{1}^{\top} \mathbf{p}^{\star}$ can be used directly as an input to Algorithm RDPC, if we replace $\nu_{l}(k)$ in Algorithm RDPC by $\nu_{l}(k) / \mathbf{1}^{\top} \mathbf{p}(k)$ for all $l$.

Recall that the convergence result in Theorem 1 requires an assumption that $\epsilon(k) \rightarrow \epsilon^{\star}$ as $k \rightarrow \infty$. For the function $\phi(\epsilon)$ in (35), further analysis can be carried out to remove this assumption and prove local asymptotic stability of Algorithm RDPC in general.

Let $\mathbf{z}(k)=\left[\mathbf{p}(k)^{\top} \mathbf{x}(k)^{\top}\right]^{\top}, \mathbf{z}^{\star}=\left[\mathbf{p}^{\star \top} \mathbf{x}^{\star \top}\right]^{\top}$ and $\Delta=$ $\delta / \mathbf{1}^{\top} \mathbf{p}^{\star}$.

Theorem 3: Consider the mapping $f$ from $\mathbf{z}(k)$ to $\mathbf{z}(k+1)$ and its Jacobian matrix $\mathbf{D}=\left.(\partial f(\mathbf{z}) / \partial \mathbf{z})\right|_{\mathbf{z}=\mathbf{z}^{\star}}$, where

$$
f(\mathbf{z})=\left[\begin{array}{c}
\left(1+\frac{\Delta}{\mathbf{p}^{\top} \mathbf{x}}\right)(\mathbf{F} \mathbf{p}+\mathbf{v}) \\
\left(1+\frac{\Delta}{\mathbf{p}^{\top} \mathbf{x}}\right) \mathbf{F}^{\top} \mathbf{x}+\mathbf{1}
\end{array}\right] .
$$

We have $\rho(\mathbf{D})=\left(1+\left(\Delta / \mathbf{p}^{\star \top} \mathbf{x}^{\star}\right)\right) \rho(\mathbf{F})$.

Furthermore, Algorithm RDPC with $\phi$ in (35) is locally asymptotically stable if and only if

$$
\rho(\mathbf{D})<1 \text {. }
$$


The local asymptotically stability property in Theorem 3 guarantees that the received SIRs of active links converge to the enhanced SIR requirements when they are slightly perturbed from the equilibrium. This is a local, rather than a global convergence result, but one that suffices in some practical cases. In these cases, for the active links, the condition that $\operatorname{SIR}_{l}(\mathbf{p}(k))$ is close to $\left(1+\epsilon^{\star}\right) \gamma_{l}$ is satisfied since the power update step in Algorithm RDPC gets executed only when $\operatorname{SIR}_{l}(\mathbf{p}(k)) \geq \gamma_{l}$, and the enhanced SIR target $\left(1+\epsilon^{\star}\right) \gamma_{l}$ is already sufficiently close to $\gamma_{l}$ since $\epsilon^{\star}$ is usually small.

\section{B. A Parameterized Family of $\phi(\epsilon)$}

Next, we turn to the speed of admitting a new user to meet its target SIR. One issue is that admission delays of new users may become intolerably large when $\epsilon$ is set to be very small. ${ }^{2}$ In any practical distributed system, new users are often given a fixed period of time for admission control. This is to avoid network instability due to a reinforced "clogging effect" when there are too many new users in a congested system [2]. In order to gain successful admission, a new feasible user (feasible as given by Lemma 1) should power up sufficiently fast to meet the SIR requirement within this period of time.

We consider the following family of $\phi_{\alpha}(\epsilon)$, parameterized by a nonnegative integer $\alpha$, for $\epsilon \in(0,1]$ :

$$
\phi_{\alpha}(\epsilon)=\delta\left(\sum_{n=1}^{\alpha}(-1)^{\alpha-n} \epsilon^{-n} / n+\log (1+1 / \epsilon)\right) .
$$

This $\phi_{\alpha}(\epsilon)$ given by (38) is strictly decreasing and also satisfies Lemma 2 (see Appendix G).

Using (38) to characterize the tradeoff between the speed of admission and energy is described as follows. First, we represent $\partial \phi_{\alpha}(\epsilon) / \partial \epsilon=-\delta /\left(\epsilon^{\alpha+1}(1+\epsilon)\right)$. This choice of $\partial \phi_{\alpha}(\epsilon) / \partial \epsilon$ provides a way to adjust the curvature of the function $\phi_{\alpha}(\epsilon)$ such that $\epsilon^{\star}$ increases as the control parameter $\alpha$, tunable by the network operator, gets larger. This implies that energy expenditure increases, but new user admission rate also increases (cf. (13) in Algorithm RDPC). Using this relationship, we have, at optimality, $\epsilon^{\star}=\left(\delta / \mathbf{1}^{\top} \boldsymbol{\nu}^{\star}\right)^{1 /(\alpha+1)}$ with $\epsilon^{\star}$ being a function that maps $\alpha \in \mathbb{Z}_{+}$to $(0,1]$ for a given $\mathbf{1}^{\top} \boldsymbol{\nu}^{\star}$. A larger $\alpha$ in (38) thus models more aggressive protection margin, but also allows new users to power up faster with increasing traffic load. Finally, we integrate the expression to get (38). Effects of adjusting $\alpha$ will be demonstrated in Experiment 3 in the next section.

\section{Interference Price and Congestion Measure}

In Algorithm RDPC, we can interpret the Lagrange multipliers $\boldsymbol{\nu}$ as "interference prices", and the sum $\mathbf{1}^{\top} \boldsymbol{\nu}$ as a congestion measure of a cellular system. As it increases, new users are admitted into the channel on a more cautionary level. In Section III, $\mathbf{1}^{\top} \boldsymbol{\nu}$ has been exploited for Algorithm RDPC design. In the following, we further discuss how $\mathbf{1}^{\top} \boldsymbol{\nu}$ intuitively quantifies the state of congestion in an interference-limited cellular network.

In the extensive literature of power control, $\rho(\mathbf{F})$ has been viewed as a congestion measure, e.g., in [17]. Congestion refers to either an increase in the number of users in the cell (thereby

\footnotetext{
${ }^{2}$ For example, in [2], an admission control heuristic is used in which even feasible new users may be forced to leave the channel if their received SIRs are still below the SIR targets after a time-out.
}

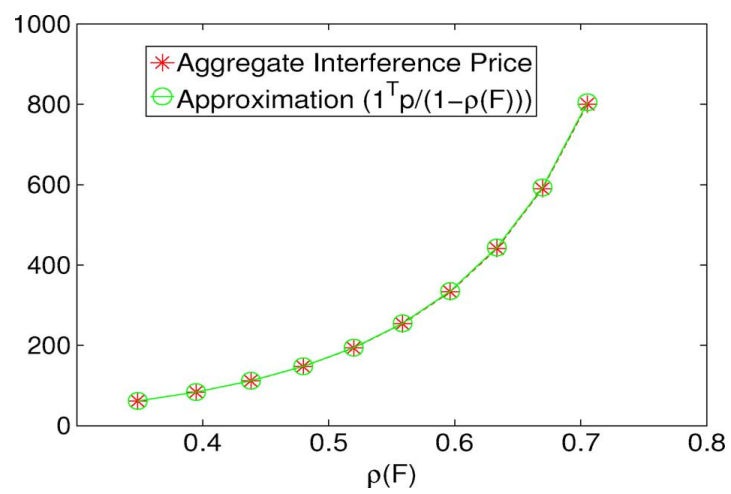

Fig. 5. A typical numerical example of using Algorithm DPC to evaluate the approximation in (39) with increasing $\rho(\mathbf{F})$.

increasing the dimension of $\mathbf{p}, \boldsymbol{\nu}$, and $\mathbf{F}$ ), or an increase in the minimum SIR requirement of any user (thereby increasing some of the elements in $\mathbf{F})$. In the former case, $\rho(\mathbf{F})$ strictly increases as shown in [17]. In the latter case, $\rho(\mathbf{F})$ strictly increases since the Perron-Frobenius eigenvalue of an irreducible nonnegative matrix is a strictly increasing function of any of its elements. Similarly, it is easy to see that $\mathbf{1}^{\top} \boldsymbol{\nu}^{\star}$ also strictly increases in these two cases.

We now connect the two measures of congestion: $1^{\top} \boldsymbol{\nu}$ from Algorithm RDPC, and $\rho(\mathbf{F})$ from the linear algebraic structures of power control problem formulations in (2) and (10).

First assume that $\rho(\mathbf{F})$ is close to, but strictly less than 1. In the case of $\epsilon=0$, Appendix I proves the following relationship:

$$
\mathbf{1}^{\top} \boldsymbol{\nu}^{\star} \approx \frac{\mathbf{1}^{\top} \mathbf{p}^{\star}}{1-\rho(\mathbf{F})} .
$$

For any $\epsilon \in(0,1]$, by considering problem (10), an approximation to $\mathbf{1}^{\top} \boldsymbol{\nu}^{\star}$ can similarly be given by

$$
\mathbf{1}^{\top} \boldsymbol{\nu}^{\star} \approx \frac{\mathbf{1}^{\top} \mathbf{p}^{\star}}{1-\left(1+\epsilon^{\star}\right) \rho(\mathbf{F})} .
$$

Using (40), the parameter $\delta$ in (35) can be related to $\rho(\mathbf{F})$ since $\delta=\mathbf{1}^{\top} \boldsymbol{\nu}^{\star} \epsilon^{\star} \approx \mathbf{1}^{\top} \mathbf{p}^{\star} \epsilon^{\star} /\left(1-\left(1+\epsilon^{\star}\right) \rho(\mathbf{F})\right)$.

Although the approximations in (39) and (40) are derived based on the assumption that $\rho(\mathbf{F})$ is close to 1 , we show numerically that the approximations are quite accurate even when $\rho(\mathbf{F})$ is much less than 1. Fig. 5 illustrates a numerical example that computes the approximation in (39) using Algorithm DPC. As shown in Fig. 5, the approximation is good for a wide range of $\rho(\mathbf{F})$ from 0.3 to 0.7 .

Lastly, the approximations in (39) and (40) can be useful in CDMA network load planning analysis for link budgets considered in [18] with the "interference price" obtained in (39) and (40) corresponding to a high network load case.

\section{NUMERICAL RESULTS}

We evaluate the performance of Algorithm RDPC with a series of five numerical experiments in progressive complexity.

\section{A. Expt. 1 (Balance Energy-Robustness Tradeoff)}

We first illustrate the ability of Algorithm RDPC to balance energy and robustness compared to Algorithm DPC and Algo- 


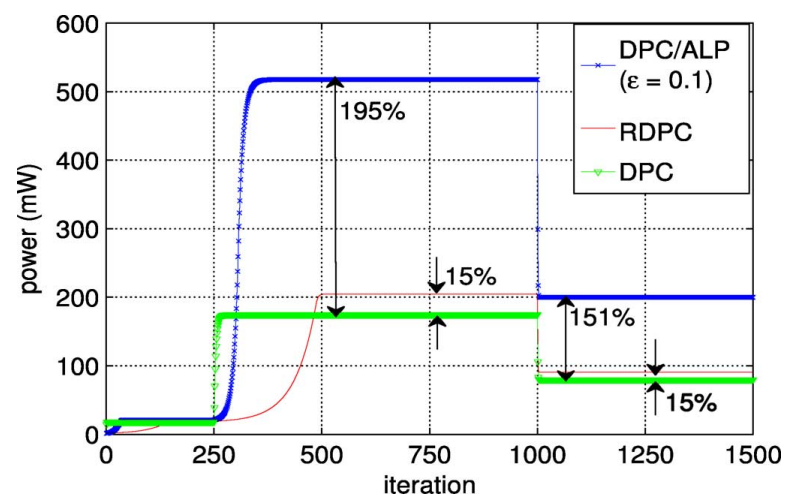

Fig. 6. Experiment 1. Evolution of the total power with DPC/ALP, RDPC and DPC algorithms. The percentage increases in extra power between (i) DPC/ALP and DPC, and (ii) RDPC and DPC at two different operating points (i.e., time slots $250-1000$ and 1000-1500) are shown.

rithm DPC/ALP. As an illustrative example, we use the channel gain matrix:

$$
\left[\begin{array}{lll}
1.000 & 0.060 & 0.070 \\
0.090 & 0.900 & 0.126 \\
0.094 & 0.064 & 0.800
\end{array}\right],
$$

noise power of $1 \mathrm{~mW}$ and the SIR targets $\gamma=(2,5,8)^{\top}$ or $(3,7,9)^{\top}$ dB. ${ }^{3}$ We assume a single cell, i.e., no interference from adjacent cells, and ignore fast fading.

Using $\phi(\epsilon)$ in (35), Algorithm RDPC is configured with $\delta$ to have an increase of at most $15 \%$ total power as compared to $\mathrm{Al}-$ gorithm DPC. During time slots 0-250, only User 1 and 2 are active. From time slot 250, User 3 becomes active. At time slot 1000 , User 1 completes data transmission, and departs from the cell leaving User 2 and 3. Fig. 6 shows that Algorithm DPC/ALP results in a total power expenditure of more than $150 \%$ at the two network operating points at time slots 250 and 1000 as compared to DPC, whereas Algorithm RDPC uses an additional extra total power of $15 \%$. Fig. 7 shows the evolution of received SIRs for Users 1, 2, and 3 when User 3 enters the channel at time slot 250. Compared to Algorithm RDPC and DPC/ALP, Algorithm DPC has the fastest convergence to the SIR targets. However, in Algorithm DPC, the received SIRs of Users 1 and 2 suffer a dip of approximately $30 \%$ and $60 \%$, respectively, at time slot 250 when User 3 enters the channel. In contrast, this does not happen with Algorithm RDPC and DPC/ALP. In summary, Algorithm RDPC offers the same amount of robustness as Algorithm DPC/ALP but with much less energy expenditure.

\section{B. Expt. 2 (Meet Total Energy Constraint $\delta)$}

As in Experiment 1, we use the SIR targets $\gamma=(3,7,9)^{\top} \mathrm{dB}$. A series of simulations are conducted with different channel gains and noise powers. We update $\epsilon$ using the $\phi(\epsilon)$ in (35) and select $\delta$ such that at most an extra total power of $33.31 \%$ is used. We observe that Algorithm RDPC converges to the global optimum that meets the percent increase specified by $\delta$. We update p, $\boldsymbol{\nu}$ and $\epsilon$ at each time slot. Fig. 8 shows the convergence behaviors.

${ }^{3} \mathrm{~A}$ typical SIR in the IS-95 CDMA system is 6 to $7 \mathrm{~dB}[10]$.
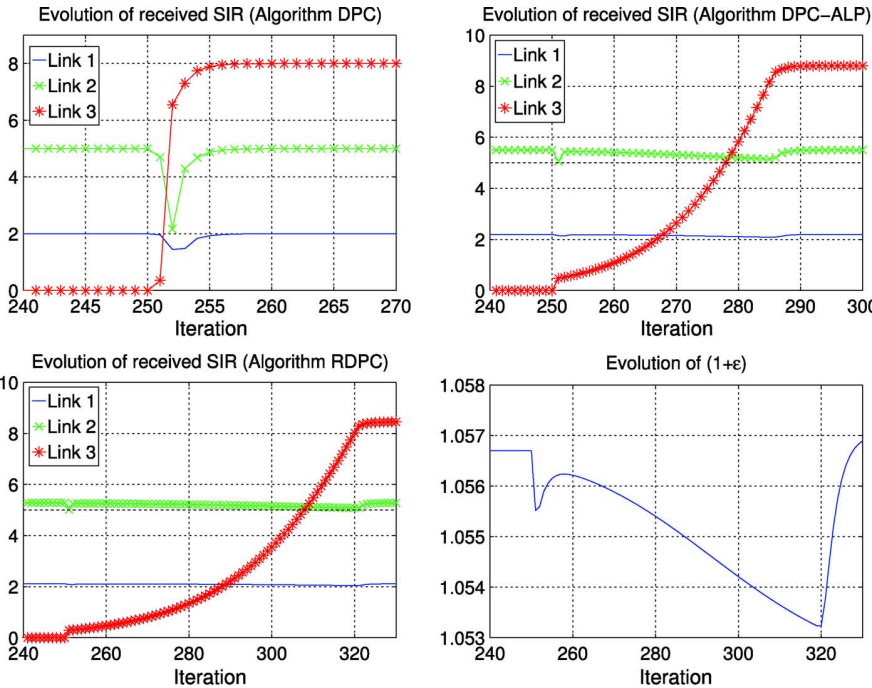

Fig. 7. Experiment 1. Evolution of received SIR when a new user enters the channel at time slot 250. The top left graph shows the SIRs for Algorithm DPC. The top right graph shows the SIRs for Algorithm DPC/ALP $(\epsilon=0.1)$. The bottom left graph shows the SIRs for Algorithm RDPC. The bottom right graph shows the evolution of $\epsilon(k)$ for Algorithm RDPC, and the $\epsilon^{\star}$ changes from 0.0566 to 0.0572 .
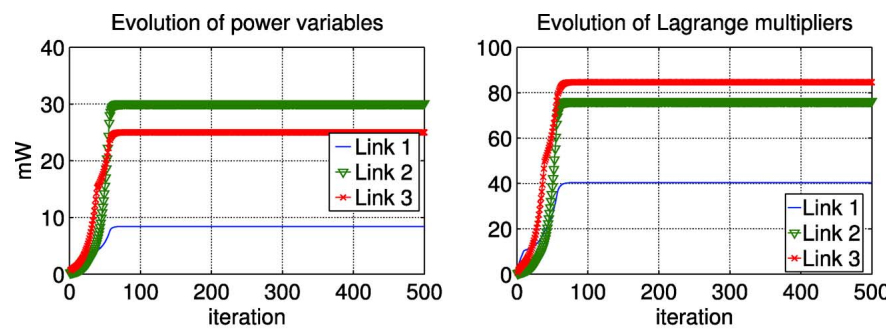

Evolution of received SIR
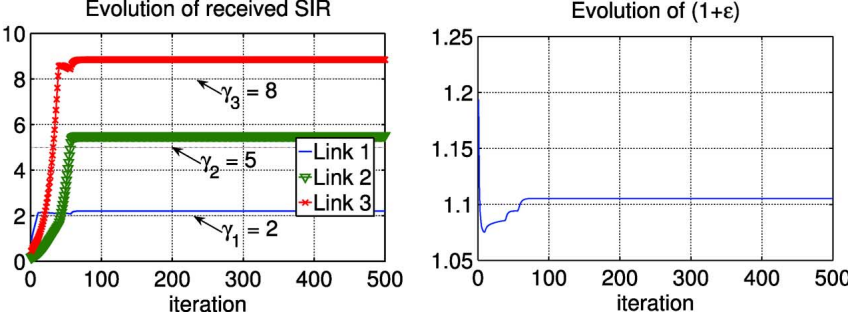

Fig. 8. Experiment 2. A typical numerical example of power and protection margin allocation using Algorithm RDPC. The top left graph shows the power variables $\mathbf{p}$. The top right graph shows the Lagrange multipliers $\nu$. The bottom left graph shows the achieved SIR with the targets, and the bottom right graph shows the parameter $\epsilon$.

\section{Expt. 3 (Accelerate Convergence)}

In this experiment, based on the $\alpha$-parameterized $\phi_{\alpha}(\epsilon)$ function in Section V-B, we propose and test a simple heuristic to achieve the goal of Experiment 2 with a faster convergence speed. We initialize a large $\alpha$ in (38), which is then decremented by 1 at every update of $\epsilon$ using $\phi_{\alpha}(\epsilon)$ in (38) until $\alpha=0$. After that, $\epsilon$ is updated using $\phi(\epsilon)$ in (35). The goal of Experiment 2 in achieving the SIR targets with a $33.3 \%$ increase in extra power is achieved after convergence. Fig. 9 shows the convergence speed of the heuristic with different initial $\alpha$ 's, and the baseline case of $\alpha=0$ (without the heuristic) is also included 
Evolution of received SIR $(\alpha=0)$

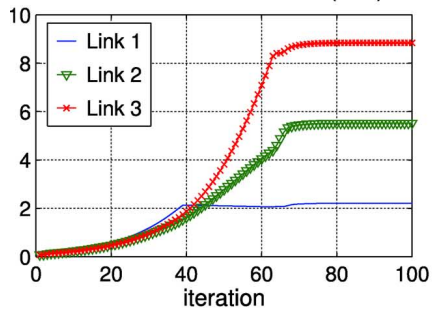

Evolution of received SIR $(\alpha=10)$

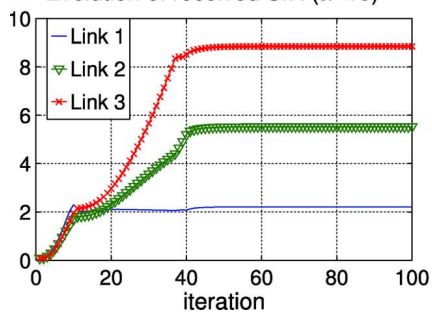

Fig. 9. Experiment 3. Accelerating convergence speed in the Algorithm RDPC using $\phi_{\alpha}(\epsilon)$ in (38) for different initial choice of $\alpha$. The top left graph shows the baseline case for $\alpha=0$, i.e., the heuristic is not used. The titles of the other graphs indicate the initial $\alpha$ used in the heuristic. As shown, in the case of $\alpha=20$, only 10 time slots are required to satisfy all the SIR targets, whereas roughly 70 time slots are required in the baseline case. The heuristic with a larger initial $\alpha$ converges much faster.

for comparison. As shown in Fig. 9, a larger $\alpha$ allows all the received SIRs to rise and stay above the SIR targets in fewer time slots than in the baseline case.

\section{Expt. 4 (Different Timescale of Updates)}

In our previous experiments, we update $(\mathbf{p}, \boldsymbol{\nu}, \epsilon)$ at the same timescale as in a primal-dual algorithm. In this experiment, we repeat Experiment 2, but the base station updates $(\boldsymbol{\nu}, \epsilon)$ at different slower timescales as compared to the timescale of power update by each user. In practice, the base station needs a relatively long time interval to measure the channel gains in order to update $(\boldsymbol{\nu}, \epsilon)$. Fig. 10 shows the convergence of the received SIRs for different timescale updates of $(\boldsymbol{\nu}, \epsilon)$. Interestingly, a slower timescale update of $(\boldsymbol{\nu}, \epsilon)$ may not lead to a slower convergence to the SIR targets $\gamma$, but only to the enhanced SIR targets $\left(1+\epsilon^{\star}\right) \boldsymbol{\gamma}$. As shown in Fig. 10, all the received SIRs rise and stay above the SIR targets $\gamma$ after the second update of $(\boldsymbol{\nu}, \epsilon)$ before converging to the enhanced SIR targets for the case of timescale (1:50), (1:100), and (1:200).

\section{E. Expt. 5 (3GPP2 Simulation With Various Network Conditions)}

The idea of providing protection against disturbance caused by new users admission can also be extended to providing protection against SIR outage induced by mobility or fading channels [19]. In this experiment, we evaluate the power control algorithms under slow fading channels.

We use a simulator that models the realistic 3GPP2 uplink evaluation network model [20]. The network model consists of 19 cells arranged in a three ring hexagonal structure. Each cell is divided into three identical 120 degree sectors for a total of 57 base station sectors, as shown in Fig. 11. The base station angular sectorization pattern is based on the commercial Decibel DB 932DG65T2E antenna, which has a 65 degree $3 \mathrm{~dB}$ bandwidth, $15 \mathrm{~dB}$ antenna gain and $20 \mathrm{~dB}$ front-to-back rejection.
Evolution of received SIR $(1: 10)$

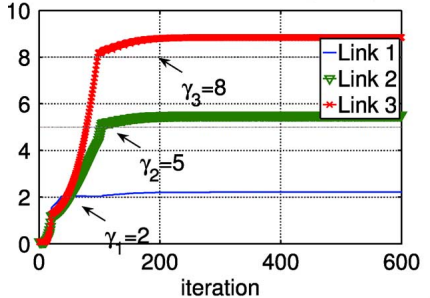

Evolution of received SIR (1:100)

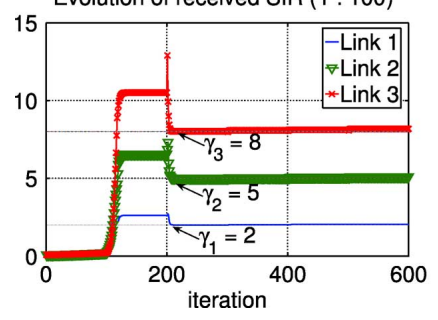

Evolution of received SIR $(1: 50)$

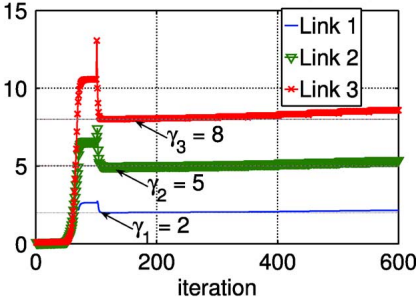

Evolution of received SIR $(1: 200)$

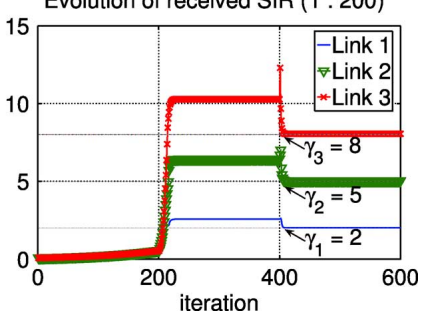

Fig. 10. Experiment 4. A typical numerical example of using Algorithm RDPC at different timescales of $(\boldsymbol{\nu}, \epsilon)$ update. A time slot on the x-axis of each graph refers to a power update iteration. The top left graph shows an update of $(\boldsymbol{\nu}, \epsilon)$ for every 10 time slots (1:10). The top right graph shows an update of $(\boldsymbol{\nu}, \epsilon)$ for every 50 time slots (1:50). The bottom left graph shows an update of $(\boldsymbol{\nu}, \epsilon)$ every 100 time slots (1:100), and the bottom right graph shows an update of $(\boldsymbol{\nu}, \epsilon)$ every 200 time slots $(1: 200)$.

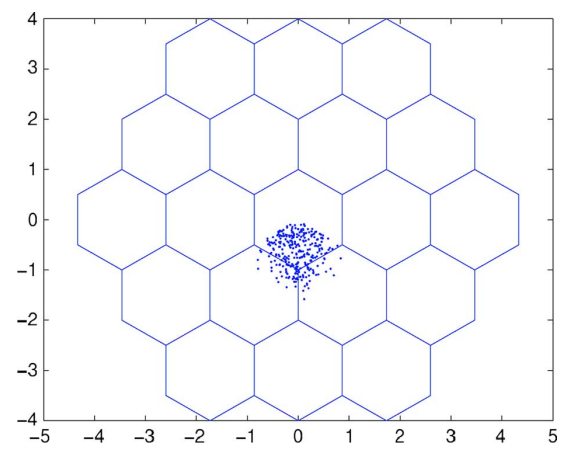

Fig. 11. A hexagonal cellular network for the uplink simulation consisting of 19 cells. Cells are three-way sectorized for a total of 57 base stations. Cell radii are normalized to unity. Path loss accounts for log-normal shadowing, and angular antenna pattern. The figure shows the locations of 300 random users connected to a base station at the lower sector of the center cell. Other users are not shown explicitly.

Each user uses a single omni-directional antenna. We adopt the pass loss model with a path loss exponent of 3.7 and log-normal shadowing standard deviation of $8.9 \mathrm{~dB}$. Users are distributed uniformly in the 19 cells and connect to the base station to which the path loss is minimum. In all our simulations, each realization of the network is initiated with 10 randomly selected users in each base station sector, with a total of 570 users. We also use a processing gain of 128 as in the IS-95 CDMA system [10]. The channel gains vary at a timescale that is 40 times slower as compared to an iteration of each algorithm.

In each of the cells, Algorithm RDPC is configured with $\phi$ in (35), where $\delta$ is set such that Algorithm RDPC uses at most 33\% more total power than Algorithm DPC. Algorithm DPC/ALP is configured with a constant $\epsilon$ that is equal to 0.1 .

First, we evaluate the network performance where users enter and exit the same channel randomly due to mobility. New users that do not reach their minimum SIR targets in 50 time slots are removed from the channel. The heuristic in Expt. 3 is used on 

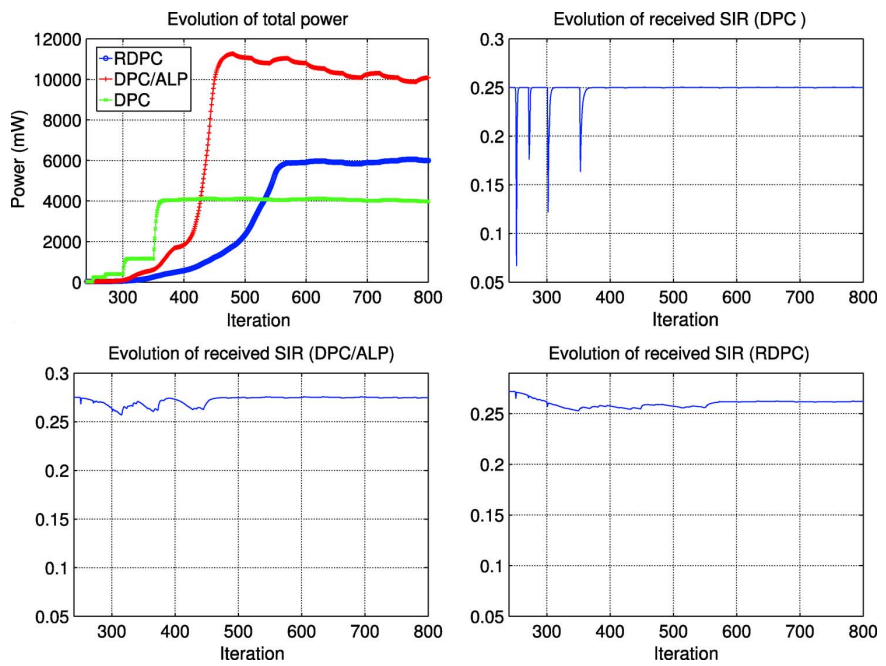

Fig. 12. Experiment 5. A typical numerical example of Experiment 5 for lognormally distributed channel gains. A time slot on the $\mathrm{x}$-axis of each graph refers to a power update iteration. Evolution of received SIR when four groups of new users enter the channel from time slot 250 to 350 . The top left graph shows the evolution of the total power. The top right graph shows the evolution of SIR for Algorithm DPC. The bottom left graph shows the evolution of SIR for Algorithm DPC/ALP $(\epsilon=0.1)$. The bottom right graph shows the evolution of SIR for Algorithm RDPC.

RDPC with initial $\alpha=10$. Fig. 12 shows a typical numerical example illustrating the total power in a particular cell and the SIR evolution of an existing user (with SIR target 0.25 ) when there are four groups of new users entering the same channel from time slot 250 to 350 , increasing the number of users from 10 to 50. As shown in Fig. 12, the user experiences SIR outage of more than $30 \%$ when Algorithm DPC is used, whereas there is no SIR outage in the case of Algorithm DPC/ALP and RDPC. On the other hand, the total power in the channel can be relatively high in the case of Algorithm DPC/ALP whereas Algorithm RDPC adapts $\epsilon$ such that there is at most 33\% increase in total power as compared to Algorithm DPC at equilibrium. We also observe from Fig. 12 that when there is a substantial number of users in the channel, the total power evolution in $\mathrm{Al}-$ gorithm DPC/ALP can have a wide range of variation, while the evolution of the total power in Algorithm RDPC is comparatively smoother. We also observe that the average percentage increase in power for each user using Algorithm RDPC is approximately equal to the percentage increase in the total power over Algorithm DPC, i.e., $33 \%$.

Next, we compare the performance of Algorithm DPC/ALP and RDPC by the average channel admission delay and the average number of users that get admitted into a particular fixed channel, which are both averaged over 10,000 random instances of the simulation. For new users accessing the channel, we implemented the time-out based voluntary drop-out scheme in [2] in conjunction with Algorithm DPC/ALP and RDPC. The voluntary drop-out scheme removes a user from the channel after a time-out period of configurable length, which is a design parameter to be optimized by testing. In all our simulations, we use a fixed time-out period of 100 time slots. The user that voluntarily drops out of the channel may access the same channel after a back-off period [2]. The heuristic in Expt. 3 is used on Algorithm RDPC with initial $\alpha=0$ and $\alpha=10$, respectively.
TABLE I

ADMISSION STATISTICS OF NEW USERS

\begin{tabular}{||c|c|c|c||}
\hline \hline & DPC/ALP & $\begin{array}{c}\text { RDPC } \\
(\alpha=0)\end{array}$ & $\begin{array}{c}\text { RDPC } \\
(\alpha=10)\end{array}$ \\
\hline \hline $\begin{array}{c}\text { Average admission } \\
\text { delay (Time slots) }\end{array}$ & 69 & 83 & 37 \\
\hline Average no. of users admitted & 15 & 19 & 28 \\
\hline
\end{tabular}

Table I shows the average channel admission delay and the average number of users that get admitted into the channel using the different algorithm settings. We observe that the network capacity (number of users supported) using Algorithm DPC/ALP can be low due to fixing $\epsilon$. Moreover, the average channel access time for Algorithm RDPC using the heuristic in Expt. 3 with $\alpha=10$ can be much lower than that in DPC/ALP. A small marginal increase in power expenditure when used appropriately can yield useful power control algorithms that are robust against different kinds of disturbance and have good performance in terms of channel admission delay and network capacity.

We conclude this experiment by mentioning that the total power expenditure for protection margin can be flexibly allocated for combating against disturbances that originate from different causes other than new user admission or mobility. For example, in a lightly loaded CDMA cell with highly mobile users that are situated far away from the base station, a larger protection margin for mobility and fading can be used as compared to a margin for guarding against admission of new users. Also, if different amount of protection margin is associated to each user (due to quality of service requirement), the results in Section III can be extended to jointly optimize all the protection margins.

\section{CONCLUSION}

Minimizing energy consumption and providing robustness of SIR targets are two important yet conflicting goals of power control in cellular networks. Built upon Algorithm DPC [1] and Algorithm DPC/ALP [2], we develop Algorithm RDPC in this paper to provide a flexible tradeoff between energy and robustness. This tradeoff is manifested both at equilibrium and during transient, and both are modulated by the shape of an objective function, e.g., through the $\alpha$-parameterized family of $\phi(\epsilon)$ proposed here. The algorithm is simple to implement, backward compatible with power control module currently in use, and contains few tunable parameters with predictive models of their effects.

A key idea behind Algorithm RDPC is to control a time-varying robustness parameter $\epsilon$ by inferring the congestion level in an interference-limited network, aided by an interference price vector $\boldsymbol{\nu}$ that iteratively updates a pair of nonlinear equations in both the primal and dual domain. Proofs establish the convergence and optimality properties of Algorithm RDPC, and numerical experiments verify its effectiveness in providing both energy management and interference management and in modulating the energy-robustness tradeoffs.

In future work, we will investigate the possibility of separating the control of tradeoff during transient from that of tradeoff at equilibrium. Another extension is to use Algorithm 
RDPC in wireless cognitive networks where the primary users are protected from the co-channel secondary users.

\section{APPENDIX}

\section{A. Proof of Lemma 1}

First, the constraints in (10): $\operatorname{SIR}_{l}(\mathbf{p}) \geq(1+\epsilon) \gamma_{l}$ for all $l$ can be rewritten as

$$
\left(1-(1+\epsilon)(\mathbf{v})_{l} / p_{l}\right) \geq(1+\epsilon)(\mathbf{F p})_{l} / p_{l}
$$

for all $l$, where $(\mathbf{v})_{l}$ denotes the $l$ th element of $\mathbf{v}$. Next, let $w_{l}=$ $q_{l} y_{l}$ for all $l$, where $\mathbf{q}$ and $\mathbf{y}$ are both strictly positive, and are the left and right eigenvectors of $\mathbf{F}$, respectively. Moreover, $\mathbf{w}$ is normalized such that $\mathbf{1}^{\top} \mathbf{W}=1$. We then raise both sides of (42) to the power of $w_{l}$ in the $l$ th constraint for all $l$, and multiply all these inequalities together to obtain a single inequality:

$$
\prod_{l}\left(1-(1+\epsilon)(\mathbf{v})_{l} / p_{l}\right)^{w_{l}} \geq(1+\epsilon) \prod_{l}\left((\mathbf{F p})_{l} / p_{l}\right)^{w_{l}}
$$

Now, it is known from [21] (cf. Theorem 3.1 in [21]) that for any irreducible nonnegative matrix $\mathbf{F}$,

$$
\prod_{l}\left((\mathbf{F p})_{l} / p_{l}\right)^{w_{l}} \geq \rho(\mathbf{F})
$$

for all $\mathbf{p}>\mathbf{0}$. This implies that $\prod_{l}\left(1-(1+\epsilon)(\mathbf{v})_{l} / p_{l}\right)^{w_{l}} \geq$ $(1+\epsilon) \rho(\mathbf{F})$. But, $\prod_{l}\left(1-(1+\epsilon)(\mathbf{v})_{l} / p_{l}\right)^{w_{l}}<1$ for all strictly positive feasible $\mathbf{p}$ and $\epsilon$, thus $(1+\epsilon) \rho(\mathbf{F})<1$.

\section{B. Proof of Lemma 2}

From the second derivative, the function $\phi\left(e^{\tilde{\epsilon}}\right)$ is convex if and only if the condition in Lemma 2 is true. The constraint set in (12) is convex since $\log \left(\operatorname{SIR}_{l}(\tilde{\mathbf{p}})\right)$ is concave in $\tilde{\mathbf{p}}$ for all $l$ (see [22]), and $\log \left(1+e^{\tilde{\epsilon}}\right)$ is convex in $\tilde{\epsilon}$.

\section{Proof of Theorem 1}

We introduce nonnegative Lagrange multipliers $\nu_{l}$ for all $l$ for the SIR constraints in (12), and write the Lagrangian

$$
\begin{aligned}
& L(\tilde{\mathbf{p}}, \tilde{\epsilon}, \boldsymbol{\nu})=\sum_{l} e^{\tilde{p}_{l}}-\sum_{l} \nu_{l}\left(\log \left(\operatorname{SIR}_{l}(\tilde{\mathbf{p}}) / \gamma_{l}\right)\right) \\
&+\phi\left(e^{\tilde{\epsilon}}\right)+\left(\sum_{l} \nu_{l}\right) \log \left(1+e^{\tilde{\epsilon}}\right) .
\end{aligned}
$$

We can minimize the above Lagrangian to obtain the Lagrange dual function $g(\boldsymbol{\nu})=\inf _{\tilde{\mathbf{p}}, \tilde{\epsilon}} L(\tilde{\mathbf{p}}, \tilde{\epsilon}, \boldsymbol{\nu})$. Next, $g(\boldsymbol{\nu})$ can be obtained by a Lagrangian decomposition:

$$
g(\boldsymbol{\nu})=g_{a l p}(\boldsymbol{\nu})+g_{d p c}(\boldsymbol{\nu}),
$$

where $g_{a l p}(\boldsymbol{\nu})$ and $g_{d p c}(\boldsymbol{\nu})$ are, respectively, the optimal values of the objective function of the following ALP subproblem:

$$
\begin{array}{ll}
\operatorname{minimize} & \phi\left(e^{\tilde{\epsilon}}\right)+\left(\sum_{l} \nu_{l}\right) \log \left(1+e^{\tilde{\epsilon}}\right) \\
\text { variables : } & \tilde{\epsilon}
\end{array}
$$

and the DPC subproblem:

$$
\begin{array}{ll}
\operatorname{minimize} & \sum_{l}\left(e^{\tilde{p}_{l}}-\nu_{l} \log \left(\operatorname{SIR}_{l}(\tilde{\mathbf{p}}) / \gamma_{l}\right)\right) \\
\text { variables : } & \tilde{p}_{\boldsymbol{l}} \quad \forall l .
\end{array}
$$

Solving (12) is equivalent to solving its Lagrange dual problem over $\boldsymbol{\nu}$ :

$$
\begin{array}{ll}
\text { maximize } & g_{a l p}(\boldsymbol{\nu})+g_{d p c}(\boldsymbol{\nu}) \\
\text { subject to } & \nu_{l} \geq 0 \quad \forall l .
\end{array}
$$

The solution of the ALP subproblem is obtained by solving the following fixed point equation for a given $\boldsymbol{\nu}$ :

$$
-\frac{\partial \phi(\epsilon)}{\partial \epsilon}(1+\epsilon)=\mathbf{1}^{\top} \boldsymbol{\nu}
$$

The subproblem DPC has a solution that is closely related to the case where $\epsilon=0$, i.e., (2). In particular, $\mathbf{p}^{\star}$ in (46) and $\nu^{\star}$ in (47) satisfy (20), which are the Karush-Kuhn-Tucker optimality conditions (cf. Theorem 2):

$$
\begin{aligned}
& \mathbf{p}^{\star}=\left(1+\epsilon^{\star}\right)\left(\mathbf{F} \mathbf{p}^{\star}+\mathbf{v}\right), \\
& \mathbf{x}^{\star}=\left(1+\epsilon^{\star}\right) \mathbf{F}^{\top} \mathbf{x}^{\star}+\mathbf{1},
\end{aligned}
$$

with

$$
\nu_{l}^{\star}=x_{l}^{\star} p_{l}^{\star} \quad \forall l .
$$

To compute $\mathbf{p}^{\star}$ in (46) and $\boldsymbol{\nu}^{\star}$ in (47), (49)-(51) are, respectively, modified to

$$
\begin{aligned}
& \mathbf{p}(k+1)=(1+\epsilon(k))(\mathbf{F} \mathbf{p}(k)+\mathbf{v}) \\
& \mathbf{x}(k+1)=(1+\epsilon(k)) \mathbf{F}^{\top} \mathbf{x}(k)+\mathbf{1}
\end{aligned}
$$

and

$$
\nu_{l}(k+1)=x_{l}(k+1) p_{l}(k+1) \quad \forall l \text {. }
$$

Using Lemma 1, we note that the fixed point of (52)-(54) must be such that $\left(1+\epsilon^{\star}\right) \rho(\mathbf{F})<1$. Assuming that $\lim \epsilon(k) \rightarrow$ $\epsilon^{\star}$ in $(48)$, and $\rho(\mathbf{F})<1 /\left(1+\epsilon^{\star}\right)$, then $\lim _{k \rightarrow \infty} \mathbf{p}(k) \rightarrow \mathbf{p}^{\star}$ and $\lim _{k \rightarrow \infty} \boldsymbol{\nu}(k) \rightarrow \boldsymbol{\nu}^{\star}$ in (52) and (54), respectively.

\section{Proof of Lemma 3}

To show (19), since $\tilde{f}^{\star}(\tilde{\mathbf{u}})$ is differentiable, for the perturbation $\tilde{\mathbf{u}}=t \mathbf{e}_{l}$ where $t$ is small, we use Taylor series expansion to obtain

$$
\tilde{f}^{\star}\left(t \mathbf{e}_{l}\right)=\tilde{f}^{\star}(\mathbf{0})+t \frac{\partial \tilde{f}^{\star}(\mathbf{0})}{\partial \tilde{u}_{l}}+o(t) .
$$

From sensitivity analysis (cf. [13, Sec. 5.6]), we have $\partial \tilde{f}^{\star}(\mathbf{0}) / \partial \tilde{u}_{l}=-\nu_{l}^{\star}$ for all $l$, which we substitute in (55). Choosing $t=\beta_{l} / 100$ yields (19).

\section{E. Proof of Theorem 2}

First, (20) is obtained by applying the Karush-Kuhn-Tucker optimality conditions to (18).

Next, by defining $x_{l}^{\star}=\nu_{l}^{\star} / p_{l}^{\star}$ for all $l$, we can rewrite (20) in matrix form as

$$
\left(\mathbf{I}-\mathbf{F}^{\top}\right) \mathbf{x}^{\star}=\mathbf{1}
$$


where 1 is a vector of appropriate dimension whose entries are all ones. Notice that we have implicitly used the fact that $\mathrm{SIR}_{l}\left(\mathbf{p}^{\star}\right)=\gamma_{l}$ for all $l$ in $(56)$.

To solve for $\boldsymbol{\nu}^{\star}$ in (20), note that $\left(\mathbf{I}-\mathbf{F}^{\top}\right) \mathbf{x}^{\star}=\mathbf{1}$ can be solved using the Perron-Frobenius theorem in a way similar to solving the power control problem in (2) [1], [2], [11]. Similarly, we propose the following algorithm, whose convergence is based on the Perron-Frobenius theorem:

$$
\mathbf{x}(k+1)=\mathbf{F}^{\top} \mathbf{x}(k)+\mathbf{1} .
$$

It converges asymptotically to $\mathbf{x}^{\star}$ if and only if $\rho(\mathbf{F})<1$, since $\rho\left(\mathbf{F}^{\top}\right)=\rho(\mathbf{F})$. Indeed, we have $\mathbf{x}(k)=\left(\mathbf{F}^{\top}\right)^{k} \mathbf{x}(0)+$ $\left[\sum_{\tau=0}^{k-1}\left(\mathbf{F}^{\top}\right)^{\tau}\right] \mathbf{1}$, which results in the limit

$$
\begin{aligned}
\lim _{k \rightarrow \infty} \mathbf{x}(k) & =\lim _{k \rightarrow \infty}\left(\mathbf{F}^{\top}\right)^{k} \mathbf{x}(0)+\lim _{k \rightarrow \infty}\left[\sum_{\tau=0}^{k-1}\left(\mathbf{F}^{\top}\right)^{\tau}\right] \mathbf{1} \\
& =\mathbf{0}+\left[\sum_{\tau=0}^{\infty}\left(\mathbf{F}^{\top}\right)^{\tau}\right] \mathbf{1} \\
& =\left(\mathbf{I}-\mathbf{F}^{\top}\right)^{-1} \mathbf{1} .
\end{aligned}
$$

Lastly, $\nu_{l}(k+1)=x_{l}(k+1) p_{l}(k+1)$ converges to $\nu_{l}^{\star}$ in (20) for all $l$ if and only if $\rho(\mathbf{F})<1$.

\section{F. Proof of Equation (32)}

From the perturbed constraint set of (17), we have $u_{l}=$ $1 /(1+\epsilon)$ for all $l$. For small $\epsilon, 1 /(1+\epsilon) \approx 1-\epsilon$, thus $u_{l} \approx 1-\epsilon$ for all $l$. Next, in (18), we let $u_{l}=e^{\tilde{u}_{l}} \approx 1+\tilde{u}_{l}$ for small (negative) $\tilde{u}_{l}$. Hence, $\tilde{u}_{l} \approx-\epsilon$ for all $l$. In Lemma 3 , we let $\beta_{l}=$ $100 \tilde{u}_{l} \approx-100 \epsilon$ for all $l$. As compared to the total power used by DPC, using Lemma 3, the increase in total power is (approximately) $\sum_{l}\left(100 \epsilon^{\star}\right) \nu_{l}^{\star} /\left(\mathbf{1}^{\top} \mathbf{p}^{\star}\right)=100\left(\mathbf{1}^{\top} \boldsymbol{\nu}^{\star} \epsilon^{\star} / \mathbf{1}^{\top} \mathbf{p}^{\star}\right)$ percent.

\section{G. Proof of (38) Satisfying Lemma 2}

We first show that $\phi_{1}(\tilde{\epsilon})=\sum_{n=1}^{\alpha}(-1)^{\alpha-n} e^{-n \tilde{\epsilon}} / n$ for all positive integer $\alpha$ is convex in $\tilde{\epsilon}$. The second derivative of $\phi_{1}(\tilde{\epsilon})$ is given by

$$
\frac{\partial^{2} \phi_{1}(\tilde{\epsilon})}{\partial \tilde{\epsilon}^{2}}=(-1)^{\alpha} \sum_{n=1}^{\alpha}(-1)^{n} n e^{-n \tilde{\epsilon}}
$$

For $\epsilon \in(0,1], \tilde{\epsilon}$ is always nonpositive. Hence, the term $n e^{-n \tilde{\epsilon}}$ is strictly increasing in $n$. For an even positive $\alpha$, there is an even number of summands in (59), which, in addition with the increasing property of $n e^{-n \tilde{\epsilon}}$, implies that the summation in (59) is positive. Thus, $\partial^{2} \phi_{1}(\tilde{\epsilon}) / \partial \tilde{\epsilon}^{2}>0$. Similarly, for an odd positive $\alpha$, the summation in (59) is negative, which together with $(-1)^{\alpha}$ gives $\partial^{2} \phi_{1}(\tilde{\epsilon}) / \partial \tilde{\epsilon}^{2}>0$. Hence, for all positive integer $\alpha, \partial^{2} \phi_{1}(\tilde{\epsilon}) / \partial \tilde{\epsilon}^{2}>0$, which implies $\phi_{1}(\tilde{\epsilon})$ is convex in $\tilde{\epsilon}$. Lastly, since $\delta>0$ and the sum of convex functions is convex, $\phi(\tilde{\epsilon})=\delta\left(\phi_{1}(\tilde{\epsilon})+\log \left(1+e^{-\tilde{\epsilon}}\right)\right)$ is thus convex in $\tilde{\epsilon}$.

Similarly, we can show that the first derivative of $\phi_{1}(\epsilon)=$ $\sum_{n=1}^{\alpha}(-1)^{\alpha-n} \epsilon^{-n} / n$ is always negative for $\epsilon \in(0,1]$. Hence, $\phi_{1}(\epsilon)$ is strictly decreasing in $\epsilon$, which implies $\phi(\epsilon)=\delta\left(\phi_{1}(\epsilon)+\right.$ $\log (1+1 / \epsilon))$ is decreasing in $\epsilon$.

\section{H. Proof of Theorem 3}

We prove the stability of Algorithm RDPC for $\phi(\epsilon)=$ $\delta \log (1+1 / \epsilon)$ by the Lyapunov's first method [23, Ch. 4], which checks the eigenvalues of the Jacobian of the nonlinear dynamical system. Let $\Delta=\delta / \mathbf{1}^{\top} \mathbf{p}^{\star}$ and $\mathbf{z}(k)=\left[\mathbf{p}(k)^{\top} \mathbf{x}(k)^{\top}\right]^{\top}$.

Consider the following nonlinear system:

$$
\mathbf{p}(k+1)=(1+\epsilon(k))(\mathbf{F} \mathbf{p}(k)+\mathbf{v})
$$

and

$$
\mathbf{x}(k+1)=(1+\epsilon(k)) \mathbf{F}^{\top} \mathbf{x}(k)+\mathbf{1}
$$

where $\epsilon(k)=\Delta / \mathbf{p}(k)^{\top} \mathbf{x}(k)$.

First, we assume that there is a fixed point to (60) and (61), and we can therefore rewrite the fixed point $\left[\mathbf{p}^{\top} \mathbf{x}^{\top}\right]^{\top}$ as

$$
\left[\begin{array}{c}
\mathbf{p} \\
\mathbf{x}
\end{array}\right]=\left[\begin{array}{c}
\left(1+\Delta / \mathbf{p}^{\top} \mathbf{x}\right)\left(\mathbf{I}-\left(1+\Delta / \mathbf{p}^{\top} \mathbf{x}\right) \mathbf{F}\right)^{-1} \mathbf{v} \\
\left(\mathbf{I}-\left(1+\Delta / \mathbf{p}^{\top} \mathbf{x}\right) \mathbf{F}^{\top}\right)^{-1} \mathbf{1}
\end{array}\right] .
$$

However, the inverse in the expression for $\mathbf{p}$ in (62) can also be expressed as

$$
\left(\mathbf{I}-\left(1+\Delta / \mathbf{p}^{\top} \mathbf{x}\right) \mathbf{F}\right)^{-1}=\sum_{i=0}^{\infty}\left(\left(1+\Delta / \mathbf{p}^{\top} \mathbf{x}\right) \mathbf{F}\right)^{i}
$$

and the series on the righthand side converges if and only if $(1+$ $\left.\Delta / \mathbf{p}^{\top} \mathbf{x}\right) \rho(\mathbf{F})<1$. Hence, the fixed point is finite if and only if $\left(1+\Delta / \mathbf{p}^{\top} \mathbf{x}\right) \rho(\mathbf{F})<1$. Similarly, this same condition holds for the inverse in the expression for $\mathbf{x}$ in (62) since $\rho\left(\mathbf{F}^{\top}\right)=\rho(\mathbf{F})$. Next, to determine whether a fixed point exists, we note that the Brouwer's fixed-point theorem [24] guarantees that there exists a fixed point $\left[\mathbf{p}^{\top} \mathbf{x}^{\top}\right]^{\top}$ since the iterative functions (60) and (61) are continuous, and $\left[\mathbf{p}^{\top} \mathbf{x}^{\top}\right]^{\top}$ lies in a compact and convex set as shown in the proof of Theorem 1 .

To show local stability around the fixed point, consider the following vector of functions:

$$
\left[\begin{array}{l}
f_{1}(\mathbf{p}, \mathbf{x}) \\
f_{2}(\mathbf{p}, \mathbf{x})
\end{array}\right]=\left[\begin{array}{l}
\left(1+\frac{\Delta}{\mathbf{p}^{\top} \mathbf{x}}\right)(\mathbf{F} \mathbf{p}+\mathbf{v}) \\
\left(1+\frac{\Delta}{\mathbf{p}^{\top} \mathbf{x}}\right) \mathbf{F}^{\top} \mathbf{x}+1
\end{array}\right] .
$$

Taking the Jacobian (denoted by D) of (64), we have

where

$$
\mathbf{D}=\left.\left[\begin{array}{ll}
\partial f_{1} / \partial \mathbf{p}^{\top} & \partial f_{1} / \partial \mathbf{x}^{\top} \\
\partial f_{2} / \partial \mathbf{p}^{\top} & \partial f_{2} / \partial \mathbf{x}^{\top}
\end{array}\right]\right|_{\mathbf{z}=\mathbf{z}^{\star}}
$$

$$
\begin{aligned}
\partial f_{1} / \partial \mathbf{p}^{\top}= & \left(1+\frac{\Delta}{\mathbf{p}^{\top} \mathbf{x}}\right) \mathbf{F}-\frac{\Delta}{\left(\mathbf{p}^{\top} \mathbf{x}\right)^{2}} \mathbf{F} \mathbf{p} \mathbf{x}^{\top} \\
& -\frac{\Delta}{\left(\mathbf{p}^{\top} \mathbf{x}\right)^{2}} \mathbf{v} \mathbf{x}^{\top}, \\
\partial f_{1} / \partial \mathbf{x}^{\top}= & -\frac{\Delta}{\left(\mathbf{p}^{\top} \mathbf{x}\right)^{2}} \mathbf{F} \mathbf{p} \mathbf{p}^{\top}-\frac{\Delta}{\left(\mathbf{p}^{\top} \mathbf{x}\right)^{2}} \mathbf{v} \mathbf{p}^{\top}, \\
\partial f_{2} / \partial \mathbf{p}^{\top}= & -\frac{\Delta}{\left(\mathbf{p}^{\top} \mathbf{x}\right)^{2}} \mathbf{F}^{\top} \mathbf{x} \mathbf{x}^{\top}
\end{aligned}
$$

and

$$
\partial f_{2} / \partial \mathbf{x}^{\top}=\left(1+\frac{\Delta}{\mathbf{p}^{\top} \mathbf{x}}\right) \mathbf{F}^{\top}-\frac{\Delta}{\left(\mathbf{p}^{\top} \mathbf{x}\right)^{2}} \mathbf{F}^{\top} \mathbf{x p}^{\top} .
$$


Hence, grouping the above expressions together, we have (omitting the superscript $\star$ for brevity):

$$
\begin{aligned}
\mathbf{D}=(1+ & \left.\frac{\Delta}{\mathbf{p}^{\top} \mathbf{x}}\right)\left[\begin{array}{cc}
\mathbf{F} & \mathbf{0} \\
\mathbf{0} & \mathbf{F}^{\top}
\end{array}\right]\left(\mathbf{I}-\frac{\frac{\Delta}{\mathbf{p}^{\top} \mathbf{x}}}{1+\frac{\Delta}{\mathbf{p}^{\top} \mathbf{x}}} \frac{1}{\mathbf{p}^{\top} \mathbf{x}}\right. \\
& \left.\times\left[\begin{array}{cc}
\mathbf{p} \mathbf{x}^{\top} & \mathbf{p} \mathbf{p}^{\top} \\
\mathbf{x} \mathbf{x}^{\top} & \mathbf{x p}
\end{array}\right]\right)-\frac{\Delta}{\left(\mathbf{p}^{\top} \mathbf{x}\right)^{2}}\left[\begin{array}{l}
\mathbf{v} \\
\mathbf{0}
\end{array}\right]\left[\begin{array}{l}
\mathbf{x} \\
\mathbf{p}
\end{array}\right]^{\top} .
\end{aligned}
$$

Let $\hat{\mathbf{D}}$ denote the matrix with entries $\left|D_{i j}\right|$. Thus, from (70) and after taking the absolute value on both sides, we have, elementwise,

$$
\hat{\mathbf{D}} \leq\left(1+\frac{\Delta}{\mathbf{p}^{\top} \mathbf{x}}\right)\left[\begin{array}{cc}
\mathbf{F} & \mathbf{0} \\
\mathbf{0} & \mathbf{F}^{\top}
\end{array}\right]+\frac{\Delta}{\left(\mathbf{p}^{\top} \mathbf{x}\right)^{2}} \mathbf{E}
$$

where

$$
\mathbf{E}=\left[\begin{array}{c}
\mathbf{F} \mathbf{p} \\
\mathbf{F}_{\mathbf{x}}^{\mathbf{x}}
\end{array}\right]\left[\begin{array}{l}
\mathbf{x} \\
\mathbf{p}
\end{array}\right]^{\top}+\left[\begin{array}{l}
\mathbf{v} \\
\mathbf{0}
\end{array}\right]\left[\begin{array}{l}
\mathbf{x} \\
\mathbf{p}
\end{array}\right]^{\top}
$$

Next, we note that

$$
\rho\left(\left(1+\Delta / \mathbf{p}^{\top} \mathbf{x}\right)\left[\begin{array}{cc}
\mathbf{F} & \mathbf{0} \\
\mathbf{0} & \mathbf{F}^{\top}
\end{array}\right]\right)=\left(\left(1+\Delta / \mathbf{p}^{\top} \mathbf{x}\right) \rho(\mathbf{F}) .\right.
$$

Moreover, when $\Delta \rightarrow 0$, we have $\rho(\hat{\mathbf{D}})=\lim _{\Delta \rightarrow 0}(1+$ $\left.\Delta / \mathbf{p}^{\top} \mathbf{x}\right) \rho(\mathbf{F})$. Since $\Delta$ can be made arbitrarily small and together with the condition for the existence of the fixed point, we have

$$
\rho(\mathbf{D}) \leq \rho(\hat{\mathbf{D}}) \leq\left(\left(1+\Delta / \mathbf{p}^{\top} \mathbf{x}\right) \rho(\mathbf{F})\right.
$$

By the Lyapunov's linearization theorem in [23, Ch. 4], the nonlinear map in (64) is locally asymptotically stable if the mapping from $\mathbf{z}(k)$ to $\mathbf{z}(k+1)$ has a Jacobian matrix $\mathbf{D}$, where $\rho(\mathbf{D})<1$. By Lemma 1 , we have $\left(\left(1+\Delta / \mathbf{p}^{\top} \mathbf{x}\right) \rho(\mathbf{F})<1\right.$, thus Theorem 3 is proved.

\section{Proof of Equations (39) and (40)}

We first derive (39). By taking the inner products of the constraint sets in (24) and (3) with $\mathbf{p}$ and $\mathbf{x}$, respectively, and noting that $\mathbf{x}^{\top} \mathbf{p}=\mathbf{1}^{\top} \boldsymbol{\nu}$, we have

$$
\mathbf{v}^{\top} \mathbf{x}+\mathbf{x}^{\top} \mathbf{F} \mathbf{p} \leq \mathbf{1}^{\top} \boldsymbol{\nu} \leq \mathbf{1}^{\top} \mathbf{p}+\mathbf{x}^{\top} \mathbf{F} \mathbf{p}
$$

which by strong duality $\left(\mathbf{1}^{\top} \mathbf{p}^{\star}=\mathbf{v}^{\top} \mathbf{x}^{\star}\right)$ leads to

$$
\mathbf{1}^{\top} \boldsymbol{\nu}^{\star}=\mathbf{1}^{\top} \mathbf{p}^{\star}+\mathbf{x}^{\star \top} \mathbf{F} \mathbf{p}^{\star}
$$

Note that (74) can also be readily obtained using Theorem 2 .

Since (24) and (3) are strictly feasible if and only if $\rho(\mathbf{F})<1$, using (74), we have

$$
\frac{\mathbf{1}^{\top} \mathbf{p}^{\star}}{\mathbf{1}^{\top} \boldsymbol{\nu}^{\star}}+\frac{\mathbf{x}^{\star \top} \mathbf{F} \mathbf{p}^{\star}}{\mathbf{1}^{\top} \boldsymbol{\nu}^{\star}}=1>\rho(\mathbf{F})
$$

where each term on the left-hand side of the inequality is positive and strictly less than 1 (since these two terms sum to 1). If $\rho(\mathbf{F})$ approaches 1 , the first and second term on the left-hand side of the inequality decreases and increases, respectively (the first term decreases since $\mathbf{x} \succeq \mathbf{1}$ and $\mathbf{x}$ increases as $\rho(\mathbf{F})$ increases, and the second term increases since the two terms must still sum to 1$)$. Hence, if $\rho(\mathbf{F})$ is close to but strictly less than 1 , we have

$$
\frac{\mathbf{x}^{\star} \mathbf{F} \mathbf{p}^{\star}}{\mathbf{1}^{\top} \boldsymbol{\nu}^{\star}} \approx \rho(\mathbf{F})=\max _{\mathbf{x} \succ \mathbf{0}} \min _{\mathbf{p} \succ \mathbf{0}} \frac{\mathbf{x}^{\top} \mathbf{F} \mathbf{p}}{\mathbf{x}^{\top} \mathbf{p}}
$$

Thus, by substituting (76) in (74), an approximation for $\mathbf{1}^{\top} \boldsymbol{\nu}^{\star}$ can be given by

$$
\mathbf{1}^{\top} \boldsymbol{\nu}^{\star} \approx \frac{\mathbf{1}^{\top} \mathbf{p}^{\star}}{1-\rho(\mathbf{F})}
$$

The approximation in (40) can also be obtained similarly.

\section{ACKNOWLEDGMENT}

The authors acknowledge helpful discussions with N. Bambos at Stanford, P. Hande at Princeton, R. Srikant at UIUC, and A. Tang at Cornell. They also thank the editor and the anonymous reviewers for their suggestions.

\section{REFERENCES}

[1] G. J. Foschini and Z. Miljanic, "A simple distributed autonomous power control algorithm and its convergence," IEEE Trans. Veh. Technol., vol. 42, no. 4, pp. 641-646, Nov. 1993.

[2] N. Bambos, C. Chen, and G. J. Pottie, "Channel access algorithms with active link protection for wireless communication networks with power control," IEEE/ACM Trans. Networking, vol. 8, no. 5, pp. 583-597, Oct. 2000.

[3] N. Bambos and S. Gitzenis, "Transmitter power control in wireless networking: Basic principles and core algorithms," in Signal Processing for Mobile Communications Handbook, M. Ibnkahla, Ed., 1st ed. Boca Raton, FL: CRC Press, 2004, ch. 23.

[4] T. ElBatt and A. Ephremides, "Joint scheduling and power control for wireless ad hoc networks," IEEE Trans. Wireless Commun., vol. 3, no. 1, pp. 74-85, Jan. 2004.

[5] G. J. Foschini and Z. Miljanic, "Distributed autonomous wireless channel assignment algorithm with power control," IEEE Trans. Veh. Technol., vol. 44, no. 3, pp. 420-429, Aug. 1995.

[6] F. Rashid-Farrokhi, K. J. R. Liu, and L. Tassiulas, "Transmit beamforming and power control for cellular wireless systems," IEEE J. Sel. Areas Commun., vol. 16, no. 8, pp. 1437-1450, Oct. 1998.

[7] D. Mitra, "An asynchronous distributed algorithm for power control in cellular radio systems," in Proc. 4th WINLAB Workshop on 3rd Generation Wireless Information Networks, Rutgers Univ., New Brunswick, NJ, 1994, pp. 249-257.

[8] P. Viswanath, V. Anantharam, and D. N. C. Tse, "Optimal sequences, power control, and user capacity of synchronous CDMA systems with linear MMSE multiuser receivers," IEEE Trans. Inf. Theory, vol. 45, no. 6, pp. 1968-1983, Sep. 1999.

[9] R. D. Yates, "A framework for uplink power control in cellular radio systems,” IEEE J. Sel. Areas Commun., vol. 13, no. 7, pp. 1341-1348, Sep. 1995.

[10] D. N. C. Tse and P. Viswanath, Fundamentals of Wireless Communication, 1st ed. Cambridge, U.K.: Cambridge Univ. Press, 2005.

[11] J. Zander, "Distributed cochannel interference control in cellular radio systems," IEEE Trans. Veh. Technol., vol. 41, no. 3, pp. 305-311, Aug. 1992.

[12] C. W. Tan, D. P. Palomar, and M. Chiang, "Exploiting hidden convexity for flexible and robust resource allocation in cellular networks," in Proc. IEEE INFOCOM, 2007, pp. 964-972.

[13] S. Boyd and L. Vanderberghe, Convex Optimization. Cambridge, U.K.: Cambridge Univ. Press, 2004.

[14] S. Koskie and Z. Gajic, "A Nash game algorithm for SIR-based power control in 3G wireless CDMA networks," IEEE/ACM Trans. Networking, vol. 13, no. 5, pp. 1-10, Oct. 2005.

[15] S. Das and H. Viswanathan, "A comparison of reverse link access schemes for next-generation cellular systems," IEEE J. Sel. Areas Commun., vol. 24, no. 3, pp. 684-692, Mar. 2006.

[16] S. Low, "A duality model of TCP and queue management algorithms," IEEE/ACM Trans. Networking, vol. 11, no. 4, pp. 525-536, Aug. 2003. 
[17] S. V. Hanly, "Congestion measures in DS-CDMA networks," IEEE Trans. Commun., vol. 47, no. 3, pp. 426-437, Mar. 1999.

[18] C. Fischer, "Load in CDMA cellular systems and its relation to the Perron root," IEEE Trans. Wireless Commun., vol. 6, no. 7, pp. 2660-2668, Jul. 2007.

[19] S. Kandukuri and S. Boyd, "Optimal power control in interference-limited fading wireless channels with outage-probability specifications," IEEE Trans. Wireless Commun., vol. 1, no. 1, pp. 46-55, Jan. 2002.

[20] Feasibility Study for Enhanced Uplink for UTRA FDD, 3GPP TR 25.896 (2004-02), Document R1-040346, V1.3.0.

[21] S. Friedland and S. Karlin, "Some inequalities for the spectral radius of non-negative matrices and applications," Duke Math. J., vol. 42, no. 3, pp. 459-490, 1975 .

[22] M. Chiang, C. W. Tan, D. P. Palomar, D. O'Neill, and D. Julian, "Power control by geometric programming," IEEE Trans. Wireless Commun., vol. 6, no. 7, pp. 2640-2651, Jul. 2007.

[23] H. K. Khalil, Nonlinear Systems, 3rd ed. Englewood Cliffs, NJ: Prentice-Hall, 2001.

[24] W. Rudin, Real and Complex Analysis. New York: McGraw-Hill, 1986.

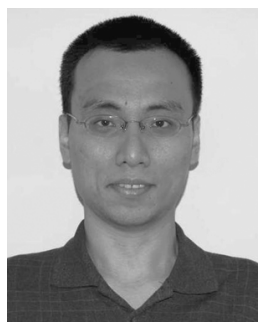

Chee Wei Tan (S'04-M'08) received the B.S. (First Class Honors) in electrical engineering from the National University of Singapore in 2002, and the M.A. and Ph.D. degrees in electrical engineering from Princeton University, Princeton, NJ, in 2006 and 2008, respectively. He is currently a Postdoctoral Scholar at the California Institute of Technology (Caltech), Pasadena, CA.

$\mathrm{He}$ was a research associate with the Advanced Networking and System Research Group at CUHK, Hong Kong, in 2004. He spent the summer of 2005 with Fraser Research Lab, Princeton, NJ. He was a Visiting Scholar at the Coordinated Science Lab of University of Illinois at Urbana-Champaign, IL, in 2007. His research interests are in wireless and broadband communications, networking, signal processing and nonlinear optimization.

He received the 2008 Princeton Wu Prize for Excellence, awarded by Princeton University.

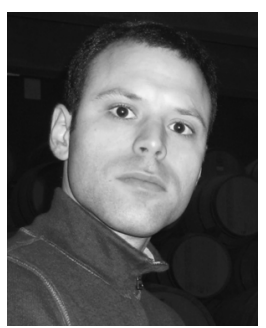

Daniel P. Palomar (S'99-M'03) received the Electrical Engineering and Ph.D. degrees (both with honors) from the Technical University of Catalonia (UPC), Barcelona, Spain, in 1998 and 2003, respectively.

Since 2006, he has been an Assistant Professor in the Department of Electronic and Computer Engineering at the Hong Kong University of Science and Technology, Hong Kong. He has held several research appointments, namely, at King's College London, London, U.K.; Technical University of
Catalonia, Barcelona; Stanford University, Stanford, CA; Telecommunications Technological Center of Catalonia, Barcelona; Royal Institute of Technology (KTH), Stockholm, Sweden; University of Rome "La Sapienza", Rome, Italy; and Princeton University, Princeton, NJ.

Dr. Palomar is an Associate Editor of IEEE TRANSACTIONS ON Signal Processing, a Guest Editor of the IEEE JouRnal of SELECTED AREAS IN COMmUniCATIONS 2008 special issue on "Game Theory in Communication Systems," and the Lead Guest Editor of the IEEE JouRnal OF SELECTED AREAS IN COMMUNICATIONS 2007 special issue on "Optimization of MIMO Transceivers for Realistic Communication Networks.” He received a 2004/06 Fulbright Research Fellowship; the 2004 Young Author Best Paper Award by the IEEE Signal Processing Society; the 2002/03 best Ph.D. prize in Information Technologies and Communications by the Technical University of Catalonia (UPC); the 2002/03 Rosina Ribalta first prize for the Best Doctoral Thesis in Information Technologies and Communications by the Epson Foundation; and the 2004 prize for the best Doctoral Thesis in Advanced Mobile Communications by the Vodafone Foundation and COIT.

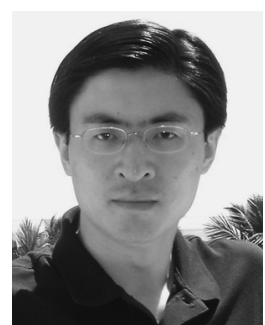

Mung Chiang (S'00-M'03-SM'08) received the B.S. (Honors) in electrical engineering and mathematics and the M.S. and Ph.D. degrees in electrical engineering from Stanford University, Stanford, NJ, in 1999, 2000, and 2003, respectively.

$\mathrm{He}$ is an Associate Professor of electrical engineering and an affiliated faculty of applied and computational mathematics and of computer science at Princeton University, Princeton, NJ. He conducts research in the areas of optimization, distributed algorithm, and stochastic models of communication networks, with applications to content distribution, broadband access, wireless networks, and the Internet.

Dr. Chiang received the CAREER Award from the National Science Foundation, Young Investigator Award from the Office of Naval Research, Howard B. Wentz Junior Faculty Award and Engineering Teaching Commendation from Princeton University, School of Engineering Terman Award from Stanford University, and was a Hertz Foundation Fellow. For his work on broadband access networks and Internet traffic engineering, he was selected for the TR35 Young Technologist Award in 2007, a list of top 35 innovators in the world under the age of 35. His work on Geometric Programming was selected by Mathematical Programming Society as one of the top three papers during 2004-2007 by young authors in the area of continuous optimization. His work on "Layering As Optimization Decomposition" became a Fast Breaking Paper in Computer Science by ISI citation in 2006. He also co-authored papers that were a IEEE INFOCOM best paper finalist and an IEEE GLOBECOM best student paper. He has served as guest or associate editor for IEEE TRANSACTIONS ON INFORMATION THEORY, IEEE/ACM TRANSACTIONS ON NETWORKING, IEEE JOURNAL OF SELECTED AREAS IN COMMUNICATIONS, IEEE TRANSACTIONS ON COMMUNICATIONS, and IEEE TRANSACTIONS ON WIRELESS COMMUNICATIONS, and Springer Journal of Optimization and Engineering, as a Program Co-Chair of the 38th Conference on Information Sciences and Systems, and as a co-editor of the new Springer book series on "Optimization and Control of Communication Systems." 